\title{
Characterization of the in-flight properties of the Planck telescope
}

\author{
J. A. Tauber ${ }^{1}$, P. H. Nielsen ${ }^{22}$, A. Martín-Polegre ${ }^{1}$, B. Crill ${ }^{18,4}$, F. Cuttaia ${ }^{15}$, K. Ganga ${ }^{2}$, J. Gudmundsson ${ }^{20,9}$, \\ W. Jones ${ }^{9}$, C. Lawrence ${ }^{18}$, P. Meinhold ${ }^{10}$, H. U. Norgaard-Nielsen ${ }^{5}$, C. A. Oxborrow ${ }^{5}$, B. Partridge ${ }^{12}$, \\ G. Roudier ${ }^{2,19,18}$, M. Sandri ${ }^{15}$, D. Scott ${ }^{7}$, L. Terenzi ${ }^{15}$, F. Villa ${ }^{15}$, J. P. Bernard ${ }^{21,3}$, C. Burigana ${ }^{14,11,16}$, \\ E. Franceschi ${ }^{15}$, H. Kurki-Suonio ${ }^{8,13}$, N. Mandolesi ${ }^{15,11}$, J. L. Puget ${ }^{17}$, and L. Toffolatti ${ }^{6,15}$
}

(Affiliations can be found after the references)

Received 3 April 2018 / Accepted 30 November 2018

\begin{abstract}
The European Space Agency's Planck satellite was launched on 14 May 2009, and surveyed the sky stably and continuously between August 2009 and October 2013. The scientific analysis of the Planck data requires understanding the optical response of its detectors, which originates partly from a physical model of the optical system. In this paper, we use in-flight measurements of planets within $\sim 1^{\circ}$ of boresight to estimate the geometrical properties of the telescope and focal plane. First, we use observed grating lobes to measure the amplitude of mechanical dimpling of the reflectors, which is caused by the hexagonal honeycomb structure of the carbon fibre reflectors. We find that the dimpling amplitude on the two reflectors is larger than expected from the ground, by $20 \%$ on the secondary and at least a factor of 2 on the primary. Second, we use the main beam shapes of 26 detectors to investigate the alignment of the various elements of the optical system, as well as the large-scale deformations of the reflectors. We develop a metric to guide an iterative fitting scheme, and are able to determine a new geometric model that fits the in-flight measurements better than the pre-flight prediction according to this metric. The new alignment model is within the mechanical tolerances expected from the ground, with some specific but minor exceptions. We find that the reflectors contain large-scale sinusoidal deformations most probably related to the mechanical supports. In spite of the better overall fit, the new model still does not fit the beam measurements at a level compatible with the needs of cosmological analysis. Nonetheless, future analysis of the Planck data would benefit from taking into account some of the features of the new model. The analysis described here exemplifies some of the limitations of in-flight retrieval of the geometry of an optical system similar to that of Planck, and provides useful information for similar efforts in future experiments.
\end{abstract}

Key words. telescopes - methods: data analysis - space vehicles: instruments

\section{Introduction}

The Planck space mission ${ }^{1}$ was launched in May 2009 and ended operations in October 2013. Between August 2009 and January 2012 it observed the sky continuously, completing five surveys of the whole sky with its two instruments: the Low Frequency Instrument (LFI) covering the range $30-70 \mathrm{GHz}$; and the High Frequency Instrument (HFI) covering the range 100-857 GHz. After January 2012, Planck continued to acquire data with the LFI for more than three additional surveys. The huge Planck data set is dedicated mainly to the study of the cosmic microwave background (CMB), with the primary aim of Planck being to measure the spatial distribution of the temperature anisotropies of the CMB with an accuracy set by astrophysical foregrounds rather than instrument performance. The first Planck maps were released in March 2013, and cosmological results based on these data were published in a series of papers (Planck Collaboration I 2014). A second and third more extended sets of Planck data, including also polarized maps, were released in 2015 and 2018, and results based on these data were published in two series of papers (Planck Collaboration I 2016, 2018).

The accuracy required by Planck science demands understanding of the optical response of each Planck detector to

\footnotetext{
1 Planck (http://wwW.esa.int/Planck) is a project of the European Space Agency (ESA), with instruments provided by two scientific Consortia funded by ESA member states and led by Principal Investigators from France and Italy, telescope reflectors provided through a collaboration between ESA and a scientific consortium led and funded by Denmark, and additional contributions from NASA (USA).
}

sub-percent level. A large effort was therefore expended on the design, development, and on-ground characterization of its optical system (Tauber et al. 2010). One of the results of this effort was a physical model of the satellite that could predict to less than $100 \mathrm{~dB}$ from peak the response of the system to radiation from the sky. This model (referred to as RFFM for Radio Frequency Flight Model) was implemented in the GRASP software developed by TICRA ${ }^{2}$, and extensively verified by measurements (Tauber et al. 2010).

In spite of the accuracy of the RFFM, it was expected that there would be some unpredicted differences between it and the actual flight performance. As a consequence, the analysis of flight data (Planck Collaboration II 2014, 2016; Planck Collaboration VI 2014; Planck Collaboration VII 2016, and references therein) relies minimally on the RFFM, using instead as much as possible on direct in-flight observations of planets (the brightest point-like objects in the Planck sky) that directly measure each detector's angular response to radiation (generally referred to as a "beam"). These measurements are described in detail in Planck Collaboration IV (2014, 2016), Planck Collaboration VII (2014), and Planck Collaboration VII (2016). A study dedicated to the estimation of the flux densities of the brightest planets is presented in Planck Collaboration Int. LII (2017) ${ }^{3}$.

\footnotetext{
2 http://www.ticra.com

3 Note that in that paper, "scanning" beams are used, which are not exactly the same as the "fsbeams" used here. More precise definitions
} of these terms can be found in Sect. 3.3. 
Nonetheless, the RFFM remains an important tool in many areas of the Planck analysis chain, as described below.

- For the LFI detectors, "hybrid" beams are synthesized products that use planet measurements where the signal-to-noise $(\mathrm{S} / \mathrm{N})$ ratio is high, and GRASP models in the lower-S/N wings of each beam. The GRASP models used in the analysis are slightly modified versions of the RFFM, whose geometrical parameters have been fit to planet measurements. In addition, the uncertainties in the beam window functions are determined by Monte Carlo variations of the aperture phase in these GRASP models, constrained to remain within the planet measurement uncertainties.

- For the HFI detectors, a similar approach as for LFI is used in that hybrid beams are synthesized, with planet measurements used at angles smaller than approximately $1^{\circ}$ from beam centre, and the RFFM (radially averaged) is used beyond.

- For both LFI and HFI detectors, the hybrid beams are used in the production of the main Planck products (maps of the full sky), as well as for the cosmological analysis (i.e., as part of the Planck Likelihood code). The hybrid beams are also used to generate realistic simulations of the mission. The RFFM itself is also used for the estimation of far-sidelobe levels, polarization response, and uncertainties related to bandpass effects.

Therefore it is important to establish the accuracy of the RFFM with regard to the in-flight performance. This paper describes such an effort. In addition, as a result of this work, a new GRASP in-flight model is developed that could be used in future analyses of the Planck data.

Finally, it is worth emphasizing that fitting of the planet measurements via GRASP models is the only way to determine the geometrical configuration of the optics in flight. From an engineering point of view, it is very useful to know to what extent the mechanical prediction is correct, and what type of mechanical deformations have not been adequately predicted.

We note that the far-sidelobe patterns (referred to as "spillover beams" in Tauber et al. 2010) are insensitive to mechanical deformations at the level discussed in this paper. They are caused by direct feedhorn illumination, with at most one reflection near the edge of a reflector. The spillover beams can be detected directly at the highest frequencies of HFI (Planck Collaboration XV 2014), and significant differences have been found in these measurements with respect to the predicted levels. These discrepancies have been attributed to manufacturing defects in the feedhorn grooves, which cannot be modelled with GRASP. The analysis that we describe in this paper has no influence on the shape of the spillover beam patterns. The RFFM consists of two main components.

- The physical and geometrical configuration of the optical system (Tauber et al. 2010), which in turn consists of:

- a telescope made of two reflectors in an offset geometry (Fig. A.1 of Tauber et al. 2010);

- a focal plane containing corrugated horns feeding each detector (Fig. 4 of Tauber et al. 2010);

- a large baffle surrounding the telescope and focal plane, which has significant optical effects on the response at very large angles from the boresight ("far sidelobes," see Tauber et al. 2010), but is not considered in this work, which is focussed on behaviour near the boresight of each beam.

- The choice of method and parameters used by GRASP to calculate each detector's response to radiation. Generally this includes physical optics for the main beams and the multireflector geometrical theory of diffraction for far sidelobes.
The methodological choices made (last item in the list above) were tuned and validated by on-ground measurements (Tauber et al. 2010) and there is no reason to believe that they should be changed. We concentrate therefore on the first item, given that the telescope geometry and reflector surface properties are potentially susceptible to mechanical and thermoelastic changes between ground and orbit, and over the lifetime of the mission.

This paper is split into two main parts, as described below.

1. The in-flight estimation of the small-scale properties of the reflector surfaces. This is based on the measurement of the properties of near sidelobes, in particular the "Ruze envelope" caused by random small-scale deformations, and "grating lobes" caused by periodic features on the reflectors (most particularly due to their honeycomb sandwich structure). These properties are detected only at the highest HFI frequencies. Results of this analysis are described in Sect. 2.

2. The in-flight estimation of the alignment between reflectors and the focal plane, and of the large-scale surface properties of each reflector. The general approach relies on the fact that we have available planet measurements of all individual detectors, each of which probes the same telescope from a different location in the focal plane and at a different frequency. A method has been developed by TICRA and ESA that exploits this fact to iteratively recover a geometrical configuration of the telescope that fits all the beam shapes optimally. The method has already been described in other papers (Jensen et al. 2010; Nielsen et al. 2011). Results of the application of this method are described in Sect. 3.

For both aspects of the analysis, we use as input measurements of planets within about $\sim 1^{\circ}$ from boresight.

In Sect. 4, we summarize the in-flight measurements of the emissivity of the Planck reflectors. In Sect. 5, we synthesize what we have learned about the in-flight geometry of the Planck optical system, and in Sect. 6, we conclude with remarks on the overall significance of this exercise described in this paper.

\section{Estimation of the small-scale structure of the reflectors}

Small-scale deformations of the optical surfaces spread power into the wings of the optical beams formed by the telescope. This is most pronounced at high frequencies. We employ the observed shapes of the beams to estimate the amplitude of periodic mechanical deformations in the primary and secondary reflectors (PR and SR). One model is analysed in Sects. 2.12.5; an alternative and simpler approach is presented in Sect. 2.6. Both provide a closer fit to the observed profile than the RFFM. We find that the measured, in-flight periodic deformations are larger than those measured on the ground.

\subsection{Effect of print-through and dimpling}

The honeycomb structure of the telescope generates two mechanical imprints on the reflector surface layers: the "printthrough" of the honeycomb core walls, which produces a distinct hexagonal pattern on the reflectors; and the so-called "dimpling" or "quilting," which results in locally concave deformations of the reflector surface around the nodes of a grid defined by the centres of the hexagonal cores. Both effects are caused by the thermo-elastic properties of the carbon-fibre honeycomb and face sheets, and of the glue that holds them together. The reflector sandwich was designed to minimize these 


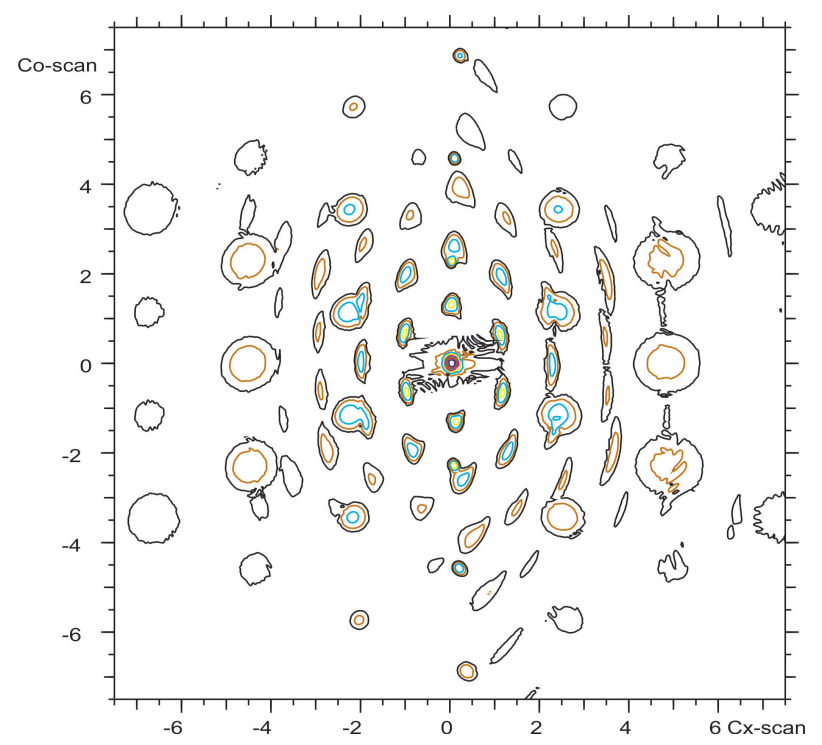

Fig. 1. Early model of dimpling, showing the typical features of grating lobes. The model is calculated at $217 \mathrm{GHz}$, and has the theoretical Planck geometry, with the addition of $10 \mu \mathrm{m}$ amplitude dimples on both primary and secondary reflectors. The contour levels are at intervals of $10 \mathrm{~dB}$ from the peak amplitude $(66.7 \mathrm{dBi})$; the outermost lobes show contours at $-13 \mathrm{dBi}$. The main lobe is in the center of the grid. The horizontal and vertical axes are in degrees, in the cross-scan and co-scan directions respectively.

mechanical signatures, but print-through and dimpling were clearly observed during ground measurements on the secondary flight reflector, both at room temperature and at the coldest temperature achieved in the test (55 K, Tauber et al. 2010). At $55 \mathrm{~K}$, the rms amplitude of print-through was measured to be $0.6 \mu \mathrm{m}$, and that of dimpling $3.6 \mu \mathrm{m}$, approximately a factor of 2 larger than at room temperature. We note that the distribution of dimpling amplitudes was close to Gaussian, with both concave and convex shapes, and only a small average in the concave direction. Individual dimples did not show a simple concave structure, rather their shapes were quite irregular. On the primary reflector, the small-scale structure was not measured at cryogenic temperature because its large size and long focal length made the interferometric measurement very complex and costly. However, based on finite-element modelling and room-temperature measurements, dimpling was expected to have lower amplitude than on the secondary due to the PR's smaller large-scale curvature.

The optical response to dimpling or print-through can be understood with a simple grating equation that describes the angular distance from beam centre, $\theta_{\mathrm{g}}$, at which incoming radiation is scattered from particular periodicities in the dimpling pattern so as to produce constructive interference. This equation is

$\sin \theta_{\mathrm{g}}=\frac{n \lambda}{Y d}$,

where $\theta_{\mathrm{g}}$ is the angular distance of the $n$ th-order lobe from the central beam peak, $\lambda$ is the wavelength of the radiation, $d$ is the grating spacing of the periodicity, and $Y$ is a magnification factor that depends on the reflector's offset with respect to the aperture plane. A typical grating pattern is shown in Fig. 1, and consists of sharp lobes located in a periodic grid around the main beam.

For the Planck reflector system, $Y \simeq 1.8$ for the secondary reflector and $Y \simeq 0.8$ for the primary reflector in the symmetry

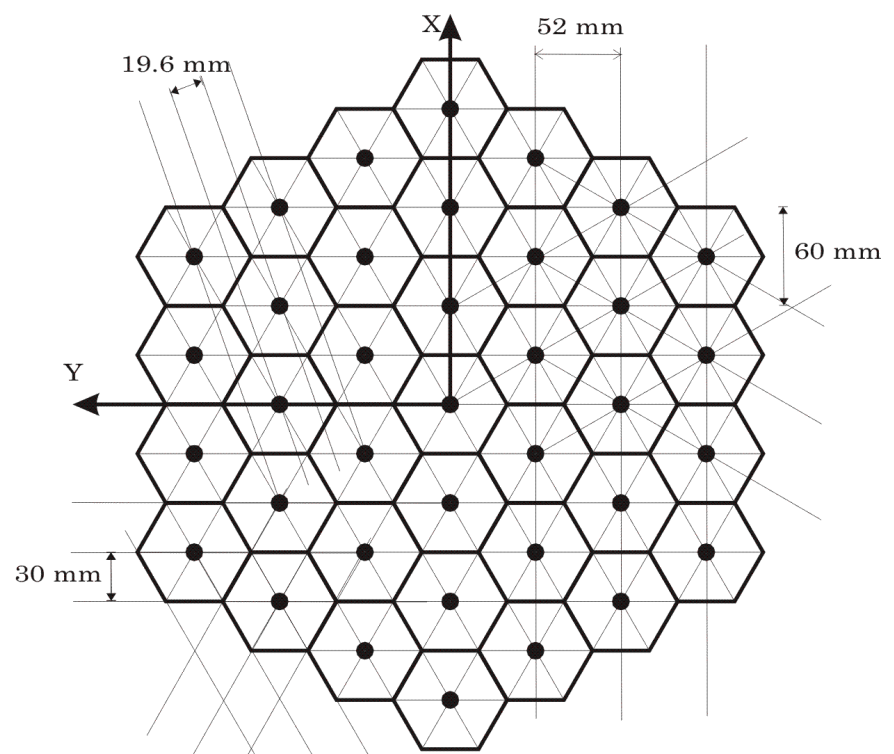

Fig. 2. Honeycomb structure of the Planck reflectors, showing the most important periodicities and the angles at which they appear.

plane (1.0 in the orthogonal plane). The most important periodicity is set by the size of the hexagonal cells $(60 \mathrm{~mm})$, which defines a grating scale of $52 \mathrm{~mm}$, appearing in the in-scan (X) direction and at $\pm 30^{\circ}$ from it (see Fig. 2). Less important periodicities are also found with size scale 30 and $19.6 \mathrm{~mm}$. Table 1 summarizes the predicted location of the most important grating lobes. Semi-analytical estimates of the strength of the lobes can be made ${ }^{4}$, yielding levels $\simeq 37,50$, and $79 \mathrm{~dB}$ below peak at $353 \mathrm{GHz}$ for a surface with constant-amplitude $10 \mu \mathrm{m}$ dimples.

It is important to remember that these predictions are only approximately correct, since the actual reflector surfaces and radiation-detection process are more complex than Eq. (1) can reflect, e.g., bandwidth effects are not taken into account. A physical simulation is therefore required to determine the actual location of the grating lobes.

The effect of dimpling was modelled using GRASP, starting in around 2000 (Nielsen 2005, 2004; Dubruel 2009). These models were used to understand the main features of the optical response to such distortions and to set requirements on the amplitude of the mechanical distortions of the reflectors. The current implementation of dimpling in the GRASP software (see, e.g., Fig. 3), consists of a 3-dimensional-cosine deformation of the reflector surface added to the nominal reflector, with peaks at the expected physical location of the dimples (the centres of the hexagonal cores) and with zeroes along the edges of the hexagonal quilting.

The full GRASP model that we build in this work uses the RFFM as a starting point. The RFFM already contains a predicted geometrical description of the telescope and focal plane at in-flight operational conditions, as determined from ground measurements (Tauber et al. 2010), to which we add further deformations of each reflector, e.g., dimples, whose amplitudes are fitted to the measurements of planets. Each time, we compute the GRASP beams for all individual detectors at a given frequency

4 Using the Ruze formula to estimate the reduction in gain due to the average phase distortion of the mechanical deformations. 
Table 1. Theoretical directions of grating lobes due to dimpling.

\begin{tabular}{|c|c|c|c|c|c|}
\hline \multirow[b]{3}{*}{ Direction } & \multirow[b]{3}{*}{ Grating scale $(\mathrm{mm})$} & \multicolumn{3}{|c|}{ Angular distance from main beam $\left(\theta_{\mathrm{g}}\right)$} & \\
\hline & & \multicolumn{2}{|c|}{$353 \mathrm{GHz}$} & \multicolumn{2}{|l|}{$857 \mathrm{GHz}$} \\
\hline & & PR & SR & PR & SR \\
\hline $\pm 30^{\circ} \pm 60^{\circ}$ & $52 \ldots \ldots \ldots$ & $1.1,2.2, \ldots$ & $0.52,1.0$ & $0.46,0.93$, & $0.21,0.42$, \\
\hline $\pm 0^{\circ} \pm 60^{\circ}$ & $30 \ldots \ldots \ldots$ & $1.9,3.9, \ldots$ & $0.90,1.8, \ldots$ & $0.80,1.60, \ldots$ & $0.37,0.74, \ldots$ \\
\hline $\pm 11^{\circ} \pm 60^{\circ}$ & $19.6 \ldots \ldots \ldots$ & $3.0, \ldots$ & $1.40, \ldots$ & $1.20, \ldots$ & $0.60,1.1, \ldots$ \\
\hline
\end{tabular}

Notes. PR is the primary reflector and SR the secondary reflector. The first two columns give the direction and length-scale of the repeating structures on the reflectors which drive each set of grating lobes. Only the first sets of lobes are indicated; additional ones appear at multiple angles.
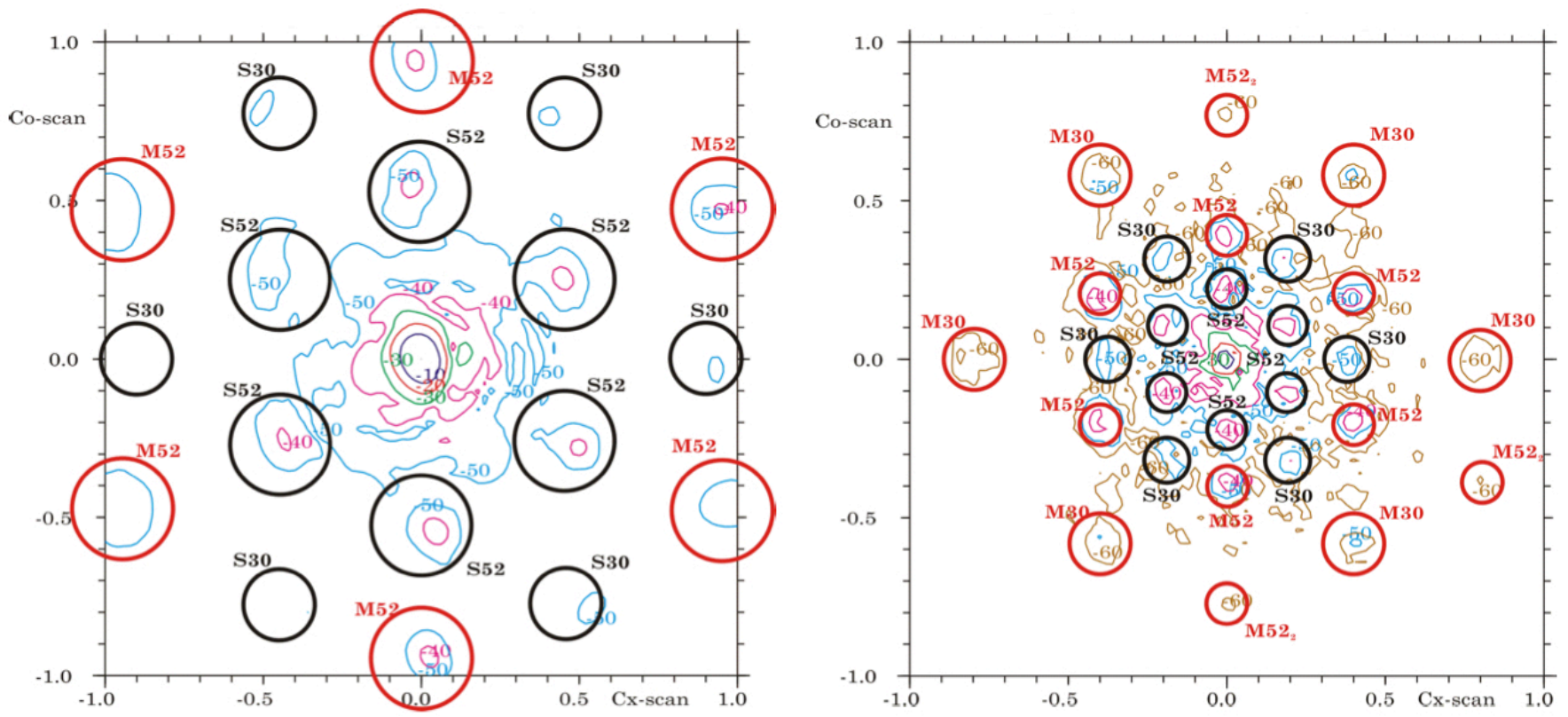

Fig. 3. GRASP model of Planck at $353 \mathrm{GHz}$ (left panel) and $857 \mathrm{GHz}$ (right panel), with circles showing the theoretical location of the main grating lobes. The model is the RFFM for individual detectors 353-1 and 857-1, to which has been added regular dimpling with $10 \mu \mathrm{m}$ amplitude. Note that the RFFM model at $857 \mathrm{GHz}$ uses a single-moded Gaussian feed, in contrast to the real multi-moded feed. The lobes are labelled according to $\mathrm{M} / \mathrm{S}$ for (Main/Secondary) and the corresponding grating size scale. The axes are in degrees and the contours are labelled in dB from peak. The peak of the main beam is suppressed for clarity.

and stack them before we compare the result to the stacked measurements (Sect. 2.2).

It is useful to emphasize that because the RFFM uses a detailed map of the secondary-reflector-surface deformations as determined before launch, it already includes dimple-like deformations. Any "new" (additional) dimpling found by fitting to in-flight data must have been caused by additional deformations. Since the primary reflector was measured on the ground with lower spatial accuracy than the secondary reflector, the RFFM uses a much less detailed mechanical model of its surface, and the dimpling levels found in flight are largely a new effect.

\subsection{Data used}

The amplitude of the sharp grating lobes was modelled before launch to be typically $40-50 \mathrm{~dB}$ below peak at $857 \mathrm{GHz}$, for a peak dimpling amplitude of $10 \mu \mathrm{m}$. Considering that the measured dimpling distortion on the reflectors was an order of magnitude lower than modelled, it was a considerable surprise when the first measurements of Jupiter (Fig. 12 of
Planck HFI Core Team 2011) at $857 \mathrm{GHz}$ yielded clear detections of grating lobes at a level $35 \mathrm{~dB}$ below peak. This high level was understood a posteriori to be due to the multi-moded nature of the feedhorns at $857 \mathrm{GHz}$, rather than to a higher-thanexpected dimple amplitude (see Sect. 2.3). Indeed, the initial GRASP models had not taken multi-moding into account, due to the difficulty and uncertainty of this type of modelling. In order to reliably compare GRASP models to the measurements, it is important to do so at frequencies where horns are single moded. Unfortunately the strength of the grating lobes reduces quickly with decreasing frequency. The best channel for this analysis therefore turns out to be $353 \mathrm{GHz}$, but even here the $\mathrm{S} / \mathrm{N}$ is low and it was necessary to wait until many observations of the major planets were acquired to achieve adequate $\mathrm{S} / \mathrm{N}$.

For this analysis, we have used all the available Planck observations of Jupiter and Saturn between 217 and $857 \mathrm{GHz}^{5}$. Maps were made of each individual detector observations near the

5 This also means that the sensitivity of the method is too low to evaluate any changes in small-scale structure that may have happened during the operational lifetime of Planck. 

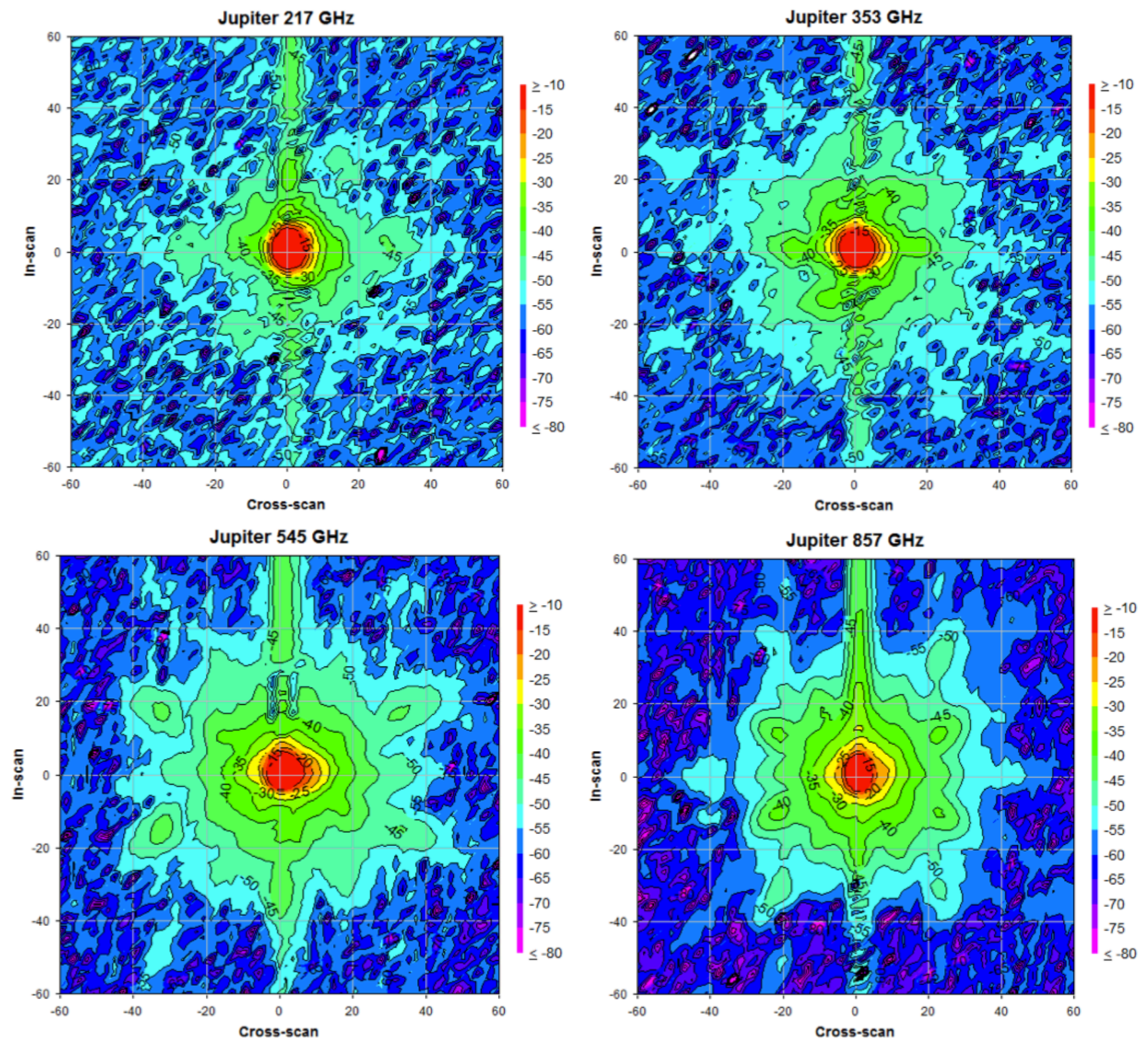

Fig. 4. Stacked observations of Jupiter for the frequencies 217-857 GHz. Here "stacked" means that all available Jupiter maps at a given frequency (from individual detectors and individual planet passes) are averaged together. The coordinate axes are in arcminutes.

two planets, using the pipeline that generated the Planck release of 2013 (Planck Collaboration VI 2014). All the available maps were stacked to increase the $\mathrm{S} / \mathrm{N}$ (meaning that all individual detector maps and all Jupiter passes were averaged), as plotted in Fig. 4.

Figure 4 shows clear detections of grating lobes at all frequencies down to $217 \mathrm{GHz}$, where with maximum stacking they are still(faintly) visible. Saturn observations show similar features, but with lowerS/N. The elongated feature in the in-scan direction at the core of the main beam is mainly a remnant of the deconvolution of the bolometer time response (Planck Collaboration VII 2016), and is masked in most of our analysis.

While the Saturn observations are believed to be completely linear, Jupiter is so bright that it is known to drive the highestfrequency bolometers into a slightly nonlinear regime around the peak of the main beam. This means that the peak Jupiter signal observed is depressed with respect to that in the wings of the maps. To overcome this problem, we have fit the wings of the
Jupiter maps to those of Saturn in the area 0.07 to 0.25 from centre (about -10 to $-35 \mathrm{~dB}$ from peak), and found that we need to renormalize the 545 (857) GHz map by 1 (2) dB to obtain consistent values. Below $545 \mathrm{GHz}$ we do not need to renormalize ${ }^{6}$. We note that the stacking process itself introduces observationdependent changes to the cores of the stacked Jupiter and Saturn maps, such that they no longer represent individual detector observations, and therefore cannot be directly compared (e.g., the FWHM of a stacked map is significantly broader than that of any single detector map). However, below $-10 \mathrm{~dB}$ from peak, the stacked maps reflect reasonably well the properties of individual detector beams. Figure 5 shows a cut through a $353 \mathrm{GHz}$ map that reveals real differences between the RFFM and the in-flight beams at levels below $-20 \mathrm{~dB}$ from peak.

6 These values are consistent with the levels of nonlinearity estimated in (Planck Collaboration Int. LII 2017), which indicate a drop by a factor of 10 in nonlinearity between 545 and $345 \mathrm{GHz}$. 


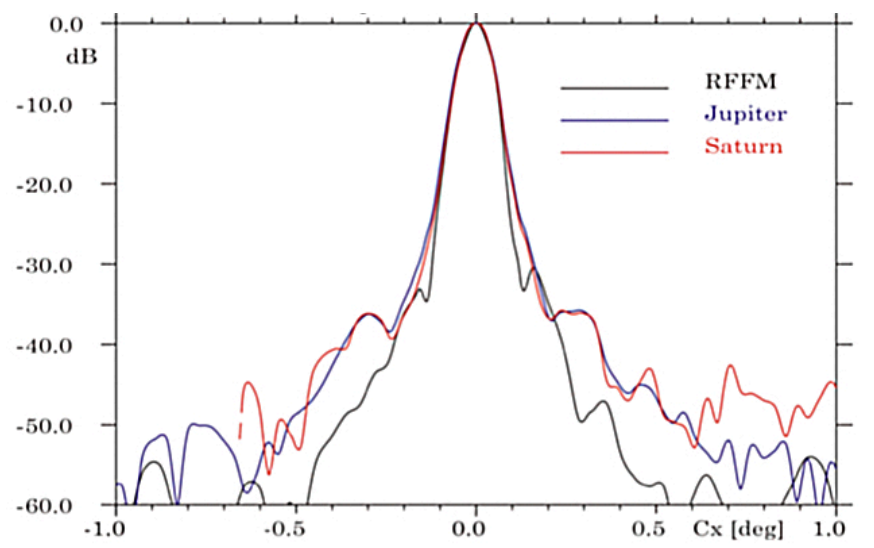

Fig. 5. Comparison of the stacked Jupiter and Saturn maps with a stacked RFFM model. The cut is in the cross-scan direction.

\subsection{Constant dimpling model}

We first attempt to fit the missing power by adding to the RFFM a simple set of constant dimples, whose only parameter is their amplitude. Close examination of Fig. 4 reveals at $353 \mathrm{GHz}$ the presence of all lobes corresponding to the $52 \mathrm{~mm}$ size scale for both primary and secondary reflectors (M52 and S52 in Fig. 3). The strength of these lobes (typically at $-50 \mathrm{~dB}$ from peak) corresponds to a mechanical amplitude of the dimples of approximately $4 \mu \mathrm{m}(2 \mu \mathrm{m})$ for the primary (secondary) reflector. Figure 6 shows the changes in the model and the improvement to the fit as these dimples are added.

This mechanical model based on $353 \mathrm{GHz}$ data is consistent with the levels of the S52 lobes at $217 \mathrm{GHz}$ (which are close to the noise floor in the stacked Jupiter map). However, at $545 \mathrm{GHz}$ the clearly-detected M52 lobes are about $5 \mathrm{~dB}$ larger than predicted. Similarly, at $857 \mathrm{GHz}$, the M52, M30, and S30 lobes are all observed to be around $10 \mathrm{~dB}$ larger than predicted. The suspicion was that this could be due to the multi-moded nature of the feedhorns at 545 and $857 \mathrm{GHz}$, which is not taken into account in the RFFM. Attempts made in the past to model the Planck multimoded horns (Murphy et al. 2010) achieved limited success in reproducing the main beam shapes. Nonetheless, a model was created with GRASP that propagates eight hybrid modes (HE01, $11,21,12,22,31,13$, and 41) through the $857 \mathrm{GHz}$ feedhorns and adds the individual beam patterns in quadrature. The results achieve the desired effect in increasing the level of the grating lobes close to the measured ones (see Fig. 7). However, considering the lack of detailed understanding of multi-moding achieved in the core of the main beam, these results should only be taken as indicative of the qualitative effects of multi-moding.

For practical reasons, our GRASP calculations are largely confined to monochromatic beams at the nominal centres of the Planck channels. However, the wideband nature of the Planck detectors may affect the results. Indeed, Eq. (1) shows that the location of the grating lobes depends on frequency. Integration over the frequency band nominally results in grating lobes that are elongated and have lower peak amplitudes than the corresponding monochromatic ones. This effect is demonstrated in Fig. 8, which shows that the peak of the grating lobes originating in the main reflector is reduced by $3.5 \mathrm{~dB}$ when the wideband nature is included. However, the grating lobes originating in the secondary reflector are much less affected than those of the primary, due to a combination of sensitivity to frequency and angular distance from peak. In order to account for these wideband effects, we need to increase the depth of the best-fit

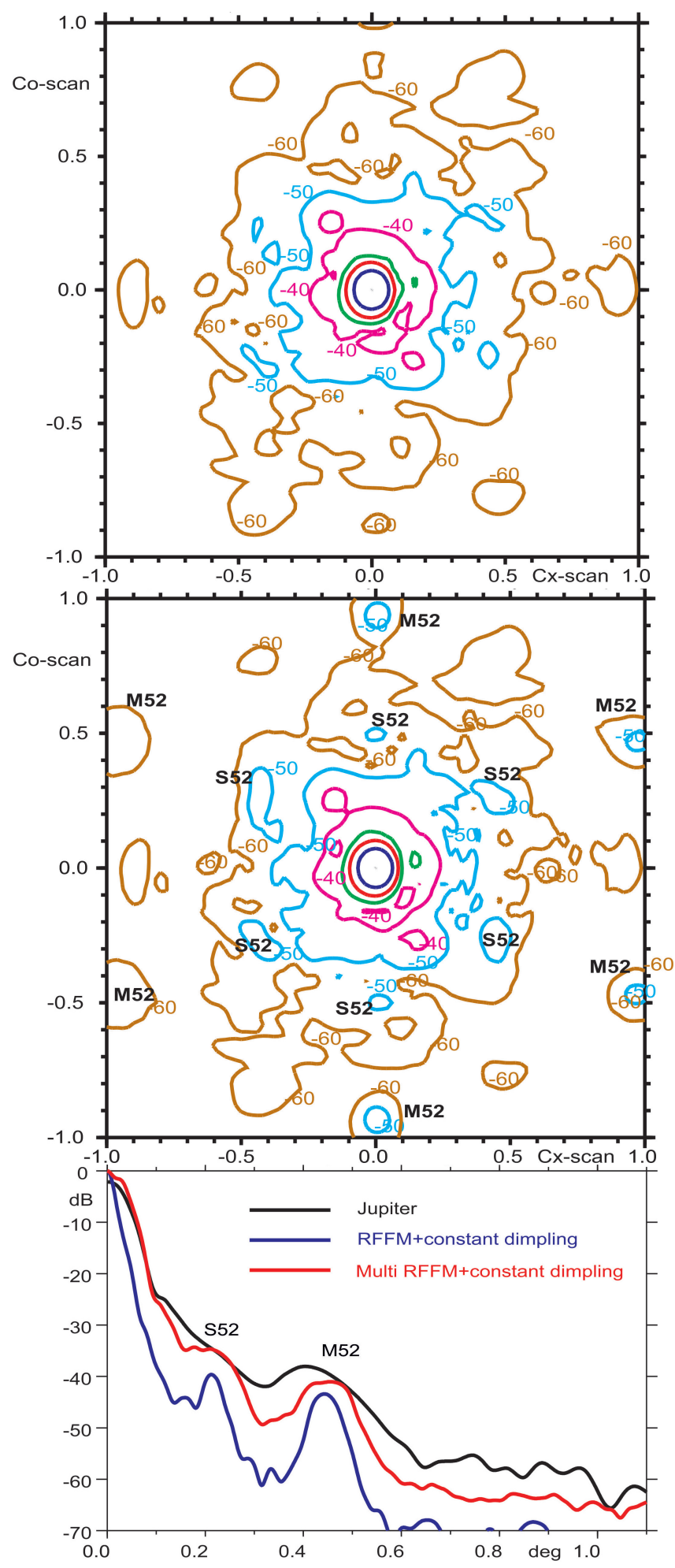

Fig. 6. Changes in the stacked model at $353 \mathrm{GHz}$, comparing the RFFM (top panel) to the RFFM, with the addition of a best-fit constantdimpling model (centre panel). The cut (bottom panel) is a stack of cuts at $\pm 16^{\circ}$ to enhance the M52 and S52 lobes. The amplitude of the S30 lobes (not shown) is hardly enhanced by the additional dimpling.

dimples on the main reflector by approximately $50 \%$, i.e., from $4 \mu \mathrm{m}$ to $6 \mu \mathrm{m}$.

The clear conclusion remains that the measured intensity of the grating lobes requires a larger amplitude of dimpling in both reflectors than predicted from the ground measurements. In 

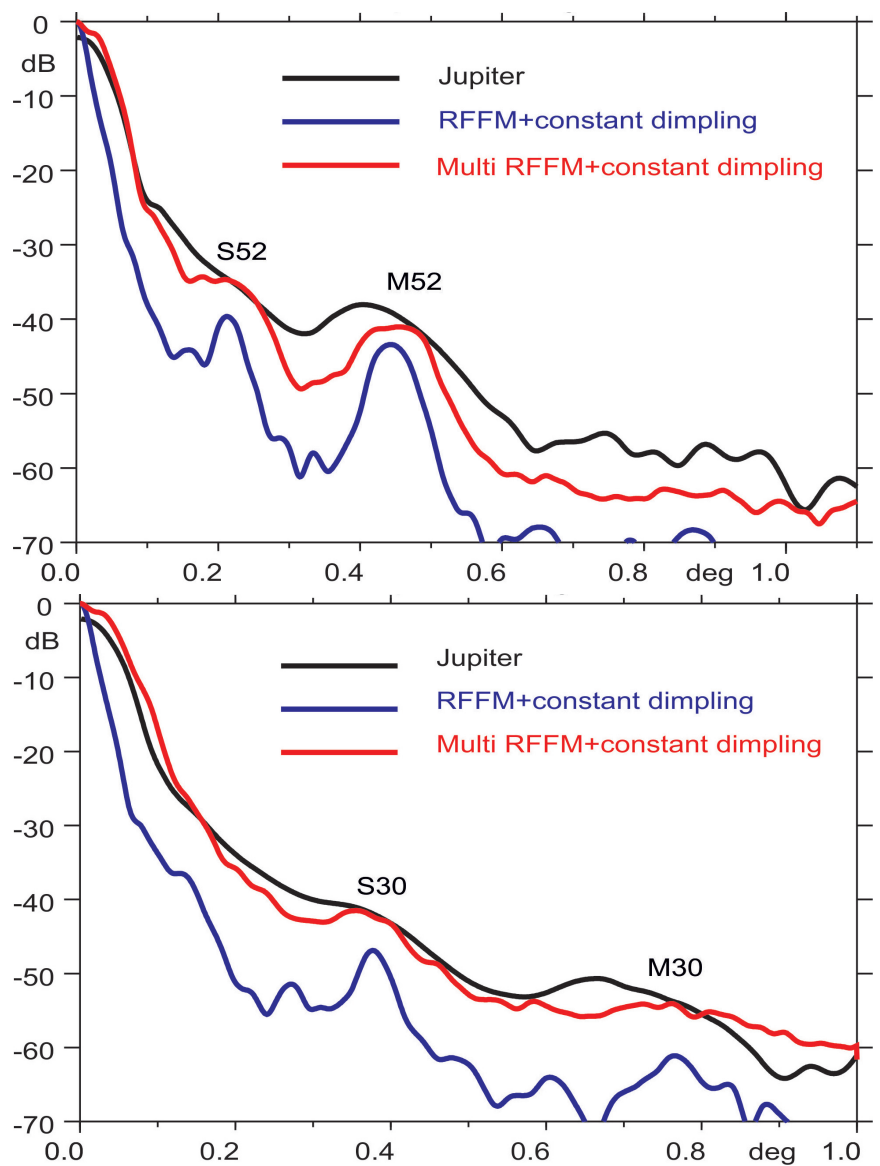

Fig. 7. Effect of multi-moding, which increases the level of grating lobes at $857 \mathrm{GHz}$, making it close to the measured ones. Two stacked cuts are shown to enhance the visibility of the main grating lobes (top panel: cuts at $\pm 26^{\circ}$, bottom panel: cuts at multiples of $60^{\circ}$ ). In blue is shown the single-moded RFFM plus constant dimpling fit at $353 \mathrm{GHz}$, and in red the equivalent multi-moded model, while the measurements on Jupiter are in black.

addition to the $3.6 \mu \mathrm{m}$ (rms) level of irregular dimpling already included in the RFFM, the SR requires an additional constant dimpling of $3 \mu \mathrm{m}$; this is not a large change. However, in the PR we were expecting levels well below $3.6 \mu \mathrm{m}$, and instead we find a constant level of around $6 \mu \mathrm{m}$. We note that the ground measurements of the SR did not reach the low temperatures achieved in flight, and the prediction was based on an extrapolation of the deformations with temperature. Another factor is the water content of the reflectors, which was certainly different in flight than in the ground measurement setting, due to the launch conditions and the fast cooldown in flight, which resulted in incomplete outgassing. These factors may explain the poor prediction. The fact remains that the mechanical behaviour of Carbon-Fibre Reinforced Polymer (CFRP) is critically dependent on the properties of its components (fibre, resin, and glue); in order to predict the mechanical behaviour to better accuracy than achieved with Planck, these properties, and that of the composite, need to be measured in environmental conditions that mimic closely the space environment.

\subsection{Sinusoidal deformations}

The best-fit model with additional constant dimpling resolves only a small part of the differences between the modelled and measured beam skirts. A six-fold pattern of lobes at a distance

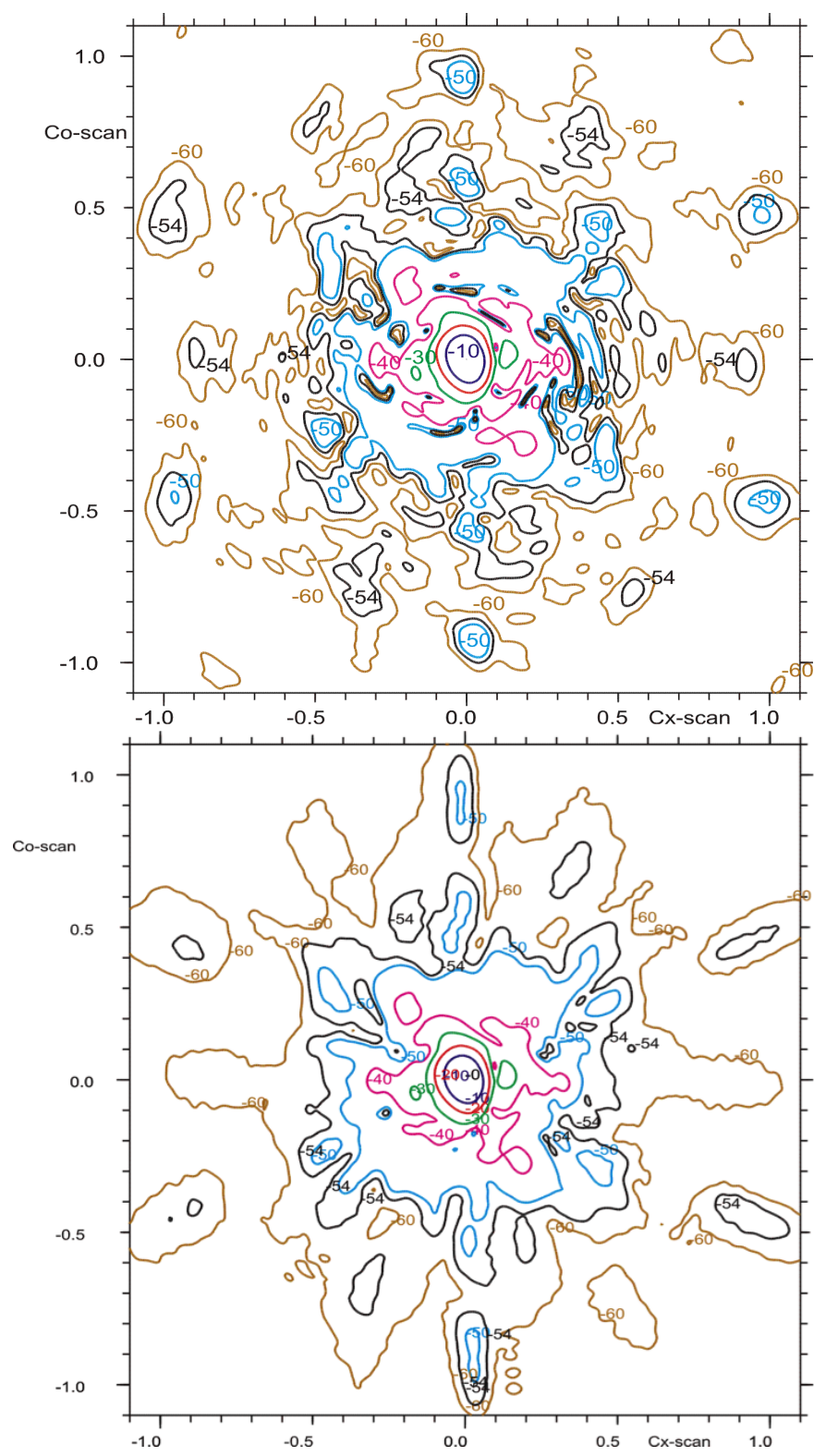

Fig. 8. Frequency dependence of the location of the grating lobes. Within the wide Planck channels, the nominal effect is to elongate the lobes radially and reduce their peak levels. This effect is particularly visible in the M52 lobes of the beam of detector 353-1a, shown in this figure. Top panel: GRASP model, based on the RFFM plus $4 \mu \mathrm{m}(2 \mu \mathrm{m})$ dimples on the primary (secondary) reflectors. Bottom panel: simulation of a wideband integration for the same geometry, represented by five GRASP models at equidistant frequencies within the band, and added in power. The peak of the M52 lobe is reduced by $3.5 \mathrm{~dB}$. Grating lobes originating in dimples in the secondary reflector are affected very little.

of 0.3 from the main beam is distinctly visible in the $353 \mathrm{GHz}$ Jupiter map, and appears as prominent shoulders in Fig. 5. This angular structure must be caused by deformations on the reflectors aligned with their symmetry (longest) axis and at $\pm 60^{\circ}$ from it, and having a mechanical periodicity of $90 \mathrm{~mm}$ on the secondary and $170 \mathrm{~mm}$ on the primary (due to the magnification). This size scale is a half-multiple of the hexagonal core dimension (see Fig. 2), and the orientations coincide with the triangle defined by the location of the reflector's isostatic mounts (ISMs; Tauber et al. 2010). Such deformations could originate in thermoelastic interaction of the ISMs and the honeycomb structure. 
The three sinusoids act to modulate the depth of the individual dimples in a regular pattern across the reflectors.

Fitting the (unequal) amplitudes of the six lobes at both 353 and $217 \mathrm{GHz}$ (where they are also clearly visible) yields amplitudes of the three sinusoidal deformations of the secondary reflector $\left(5,4\right.$, and $0 \mu \mathrm{m}$ in the $0^{\circ}, 60^{\circ}$, and $120^{\circ}$ planes, respetively), resulting in a remarkably close fit of the resulting pattern (see Fig. 9). Similar deformations can be fit at $353 \mathrm{GHz}$ to yield much lower amplitudes $(<1.2 \mu \mathrm{m}$ in all three planes); at $217 \mathrm{GHz}$ the lobes caused by these features are below the noise floor. The corresponding features at higher frequencies are not clearly observed, since they appear at smaller distances from beam centre $0.2\left(0^{\circ} .44\right)$ and $0.12\left(0^{\circ}\right)$ due to the secondary (primary) at 545 and $847 \mathrm{GHz}$ ) and - even after amplification by multi-moding - at a level similar or below the skirt of the main beam; there are hints of their presence in the measured maps, but they cannot be used to infer the amplitude of potential sinusoidal deformations of the primary.

\subsection{Ruze-like deformations}

Figures 5 and 9 show that the dimpling+sinusoidal deformation model at $353 \mathrm{GHz}$ still under-predicts a significant amount of power in the measured patterns around 0.1 from centre (between -20 and $-30 \mathrm{~dB}$ from peak). None of the dimpling-related features included so far are able to scatter significant power into this angular range, though it is affected by the large-angular-scale deformations and realignments analysed in Sect. 3.3. We investigate here whether scattering from random-amplitude deformations spread across the reflectors could account for these differences. Such deformations can be modelled using the Ruze formalism. Indeed, the Ruze equation implies that to significantly affect this angular region, the correlation length of mechanical deformations should be larger than $180 \mathrm{~mm}$ $(420 \mathrm{~mm})$ on the secondary (primary), and their rms amplitude should be larger than around $14 \mu \mathrm{m}(19 \mu \mathrm{m})$. It is very unlikely that the reflectors contain mechanical deformations of a periodic or random nature with these characteristics; furthermore Ruze-like envelopes for randomized deformations would lead to degree-scale skirts in the patterns, which should be far stronger than the measurements indicate. Ruze-like envelopes could explain the high skirts seen at 545 and $857 \mathrm{GHz}$ that extend to degree scales, although they would require fairly high rms amplitudes (of order $10 \mu \mathrm{m}$ ). However, we have already seen (in Fig. 7) that multi-moding can readily account for these high skirts, and this is the most plausible explanation.

Overall we do not find significant evidence for the existence of random or periodic deformation of the reflectors at size scales significantly larger than that of the hexagonal cores. In Sect. 3 we will investigate if there are large-scale deformations of the reflectors or misalignments of the system that could improve the fit of the $353 \mathrm{GHz}$ model at around $0^{\circ} .1$ from beam centre.

\subsection{Scaled dimpling}

The physical model of the reflectors built so far in the analysis consists of the RFFM plus constant dimpling and sinusoidal deformations on each reflector (see Table 2). As mentioned earlier, the RFFM already includes small-scale structure in the secondary reflector, whereas in the primary it does not. A potentially more reasonable physical model would be to substitute the "new" (additional) dimples on the secondary reflector with a linear enhancement of the already-existing deformations in the RFFM SR. We have investigated such a model and the resulting
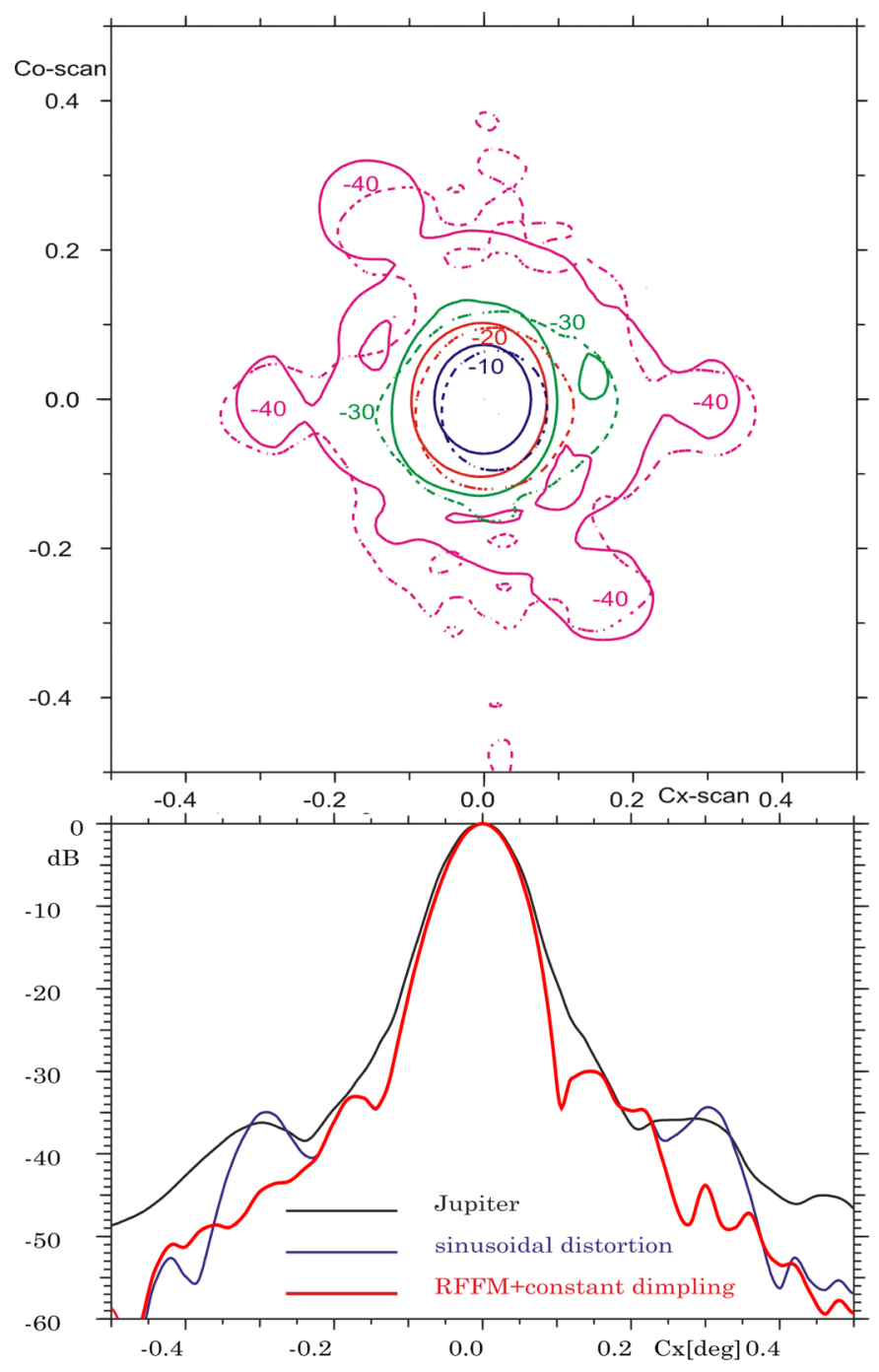

Fig. 9. Model with three sinusoidal deformations of the subreflector at $353 \mathrm{GHz}$. This yields a good fit to the lobes located $0.3^{\circ}$ from beam centre. We compare here the RFFM + constant dimpling model with and without the addition of these deformations. The top panel shows the measured Jupiter map in dashed contours.

best-fit scaling parameter for the secondary reflector deformations is 1.2 , an interestingly low value that fits well with the combination of the dimple level found in the constant-dimple model with the average dimple depth measured on the ground. A good feature of this alternative model is that the enhanced RFFM deformations also generate some of the lobes that were previously fit with sinusoidal deformations. This implies that lower amplitudes of the latter are required on the subreflector in the alternative model.

The two models retrieved are compared to the measurements in Fig. 10. Overall, the alternative "Model 2" is preferred over the initial one, since: (a) it requires fewer (and possibly more physically reasonable) additional deformations; and (b) it fits the measured Jupiter maps better. We illustrate it in Figs. 11 and 12

\subsection{Summary}

A summary of the two geometrical models retrieved for the reflector surfaces at small scales is presented in Table 2. As described in the previous section, Model 2 is the one that we prefer. 
Table 2. Characteristics of reflector surfaces fitted to grating lobes.

\begin{tabular}{|c|c|c|c|c|}
\hline \multirow[b]{2}{*}{ Characteristic } & \multicolumn{2}{|l|}{ Model 1} & \multicolumn{2}{|l|}{ Model 2} \\
\hline & PR & SR & PR & SR \\
\hline $\begin{array}{l}\text { Small-scale deformations...................... } \\
\text { Constant dimpling (peak } \mu \mathrm{m}) \ldots \ldots \ldots \ldots \\
\text { Sinusoidal deformations }(\mu \mathrm{m})\left(0^{\circ}, 60^{\circ}, 120^{\circ}\right) \ldots\end{array}$ & $\begin{array}{c}\text { RFFM } \\
-6 \\
(<1.2,<1.2,<1.2)\end{array}$ & $\begin{array}{l}\text { RFFM } \\
-2 \\
(5,4,0)\end{array}$ & $\begin{array}{c}\text { RFFM } \\
-6 \\
(<1.2,<1.2,<1.2)\end{array}$ & $\begin{array}{c}1.2 \times \text { RFFM } \\
0 \\
(2,0,0)\end{array}$ \\
\hline
\end{tabular}

Notes. On the secondary reflector, the "Constant dimpling" is in addition to the measured features.

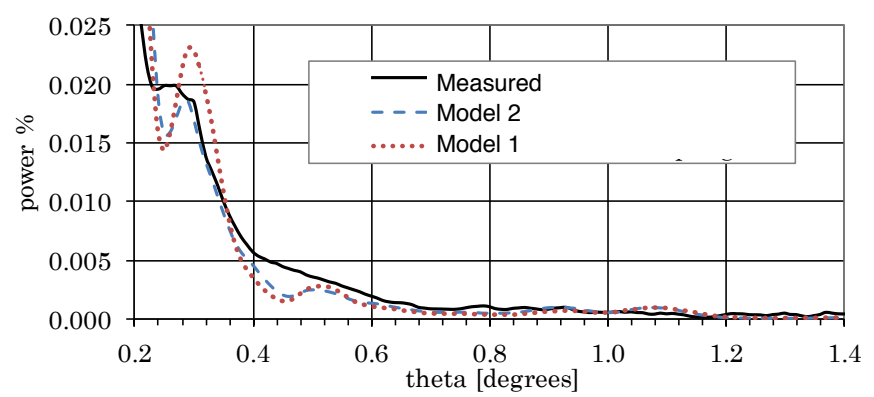

Fig. 10. Comparison of the two retrieved models (Model 1 and 2, see Table 2) against the measurements. The plot shows the difference in power between the measured Jupiter map (solid black line) and the two models, integrated in 0.01 radial bins. The measured pattern is normalized such that the total power within the region $\theta<1.4$ is equal to that in the model pattern; in addition an offset is added to account for its noise floor. Use of a mask covering the areas affected by residual time response effects has a negligible effect.

For the beam representation used in Planck analysis, it is of interest to understand how much power is involved in the grating lobes studied here. Table 3 is based on the stacked model at $353 \mathrm{GHz}$ (see Fig. 12) and gives an indication of the total power involved in some of the main grating features.

For scientific analysis (e.g., mapmaking for CMB work), the response of individual detectors is more relevant than that of the stack, so we provide here radial plots and radially binned powers using Model 2 for two representative detectors, 217-1a (Fig. 13 and Table 5) and 353-1a (Fig. 13 and Table 4). It is also interesting to estimate the power in individual grating lobes, which is presented in Table 6. Differences between Tables 3 and 4 can be taken as indications of the uncertainty in the determination of power levels. Note that Tables 3 and 4 approximately include the effect of integration over the bandwidth of each channel.

\section{Estimation of the telescope geometry using multi-beam information}

We turn now to larger-scale deformations and offsets that affect the beam shape at smaller angles from the optical axis. These include both the relative location of each optical element, and large-scale surface deformations of the reflectors. Analysis of the in-flight beams made from observations of planets have allowed us, to some degree, to refine the geometrical model for Planck optics. We emphasize once again that the results of the tests described in this section were not used in the scientific analysis of Planck data.

We note that the range of angles that we fit in this section does not overlap with the much larger angles investigated in Sect. 2, in which the dimpling-induced grating lobes appear. For example, at $353 \mathrm{GHz}$, the grating lobes appear at angles larger
Table 3. Total power in radial bins for the stacked Model 2 at $353 \mathrm{GHz}$.

\begin{tabular}{ccc}
\hline \hline Radial bin & Grating lobes & Power $(\%)$ \\
\hline $0.28-0.40 \ldots \ldots$ & Sinusoidal (SR) & 0.14 \\
$0.40-0.70 \ldots \ldots$ & S52 & 0.11 \\
$0.70-0.80 \ldots \ldots$ & $\ldots$ & 0.02 \\
$0.80-1.10 \ldots \ldots$ & M52 + S30 & 0.06 \\
\hline
\end{tabular}

than 0.3 , whereas the main beam fit is made out to angles 0.15 . The analysis of Sect. 2 does not affect that of Sect. 3 and vice versa; the best-fit deformations found in each can simply be added in the GRASP model.

\subsection{The method}

The method used in this work was developed by TICRA for ESA and described in Jensen et al. (2010). The principle consists of fitting geometrical models of an array of beam patterns iteratively to measurements, varying geometrical parameters (alignment and surface shape) until convergence is achieved. Both the shapes of individual beams and the relative distribution of beams on the sky (the "footprint") contribute information to the process. The method is quite suited to the case of Planck, where the wide frequency range $(30-857 \mathrm{GHz})$ and the large field of view $(55$ feedhorns spread over $9^{\circ} \times 8^{\circ}$ on the sky) probe a large range of beam shapes.

The fit is carried out by a modified version of the software package $\operatorname{POS}^{7}$ (which is usually used in the reverse direction, namely to force patterns to a desired shape) and the calculation of patterns using GRASP. The merit function that is minimized (which we refer to in the rest of this article as the "variance," $\delta$ ) is the quadratic difference of the logarithms of the modelled and measured beams above a given signal threshold, weighted by the $\mathrm{S} / \mathrm{N}$ of each sample:

$\delta=\sqrt{\frac{\sum_{b=1}^{N_{\mathrm{b}}} \frac{1}{N_{\mathrm{b}}} \sum_{i=1}^{N_{\mathrm{s}}^{\mathrm{b}}} f_{\mathrm{bi}}^{2}}{\sum_{b=1}^{N_{\mathrm{b}}} \frac{w_{b}^{2}}{N_{\mathrm{b}}} \sum_{i=1}^{N_{\mathrm{s}}^{\mathrm{b}}} w_{i}^{2}}}$.

Here $N_{\mathrm{b}}$ is the number of beams included, $N_{\mathrm{s}}^{\mathrm{b}}$ is the number of measured samples used for each beam, and

$f_{\mathrm{bi}}(x)=w_{b} w_{i}\left(d_{\mathrm{bi}}-\bar{d}_{\mathrm{bi}}(x)-\mu_{b}\right)=w_{b} w_{i}\left(\Delta_{i}(x)-\mu_{b}\right)$,

where $d_{\mathrm{bi}}$ is the vector of measured samples (in $\mathrm{dB}$ from peak) for beam $b$, and $\bar{d}_{\mathrm{bi}}(x)$ is the modelled pattern that depends on a set of geometrical parameters $x$. Since we cannot measure in flight the total power contained in each beam, the measured

7 http://www.ticra.com 

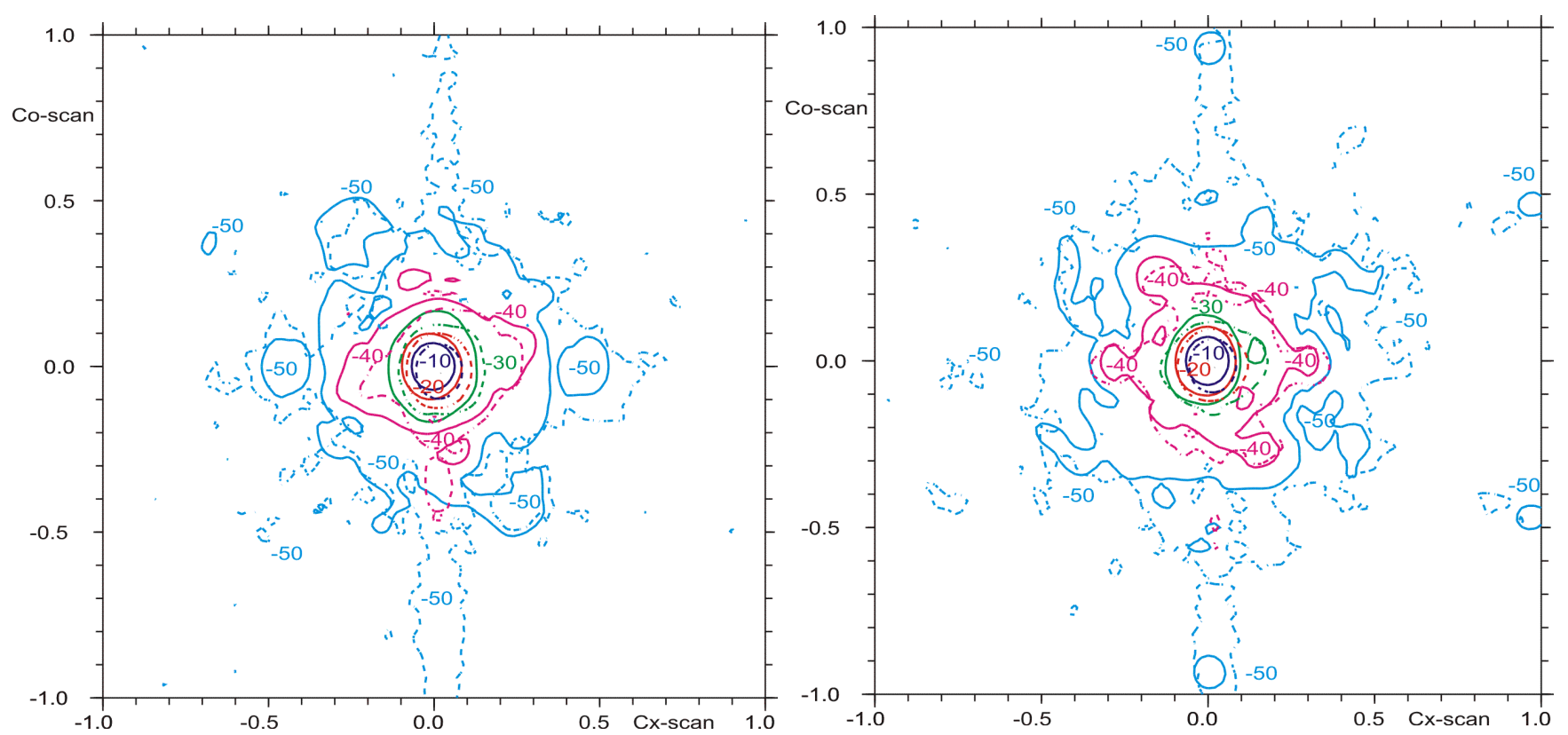

Fig. 11. Model 2, showing the model (full line) and measurements (dashed line) at $217 \mathrm{GHz}$ (left panel) and $353 \mathrm{GHz}$ (right panel). The coordinate axes are in degrees.

Table 4. Total power in radial bins for Model 2 and detector 353-1.

\begin{tabular}{ccc}
\hline \hline Radial bin & Grating lobes & Power $(\%)$ \\
\hline $0.28-0.40 \ldots \ldots$ & Sinusoidal (SR) & 0.18 \\
$0.40-0.70 \ldots \ldots$ & S52 & 0.06 \\
$0.70-0.80 \ldots \ldots$ & $\ldots$ & 0.01 \\
$0.80-1.10 \ldots \ldots$ & M52 + S30 & 0.03 \\
\hline
\end{tabular}

beams need to be re-normalized. This is done by means of an offset determined from the data themselves:

$\mu_{b}=\frac{\sum_{i=1}^{N_{s}^{b}} w_{i}^{2} \Delta_{i}}{\sum_{i=1}^{N_{s}^{b}} w_{i}^{2}}$.

Each measured sample is weighted $\left(w_{i}\right)$ according to its $\mathrm{S} / \mathrm{N}$, and each beam is given a weight $\left(w_{b}\right)$ within the ensemble, which depends on the signal threshold used for that beam and the confidence in the quality of the measured beam.

The method was initially tested on simulations of the Planck optical system (Jensen et al. 2010), where it was shown that it could recover a $1 \mathrm{~mm}$ defocus of the system to $0.01 \mathrm{~mm}$ accuracy. Translations along the focal plane are recovered to approximately $0.1 \mathrm{~mm}$, and rotations of a few arcminutes are recovered to about 0.2 arcmin accuracy. The amplitude of loworder reflector distortions (modelled as Zernike polynomials) could be recovered to $0.01 \mathrm{~mm}$ accuracy.

It is interesting to note that during the first applications of the method to real in-flight Planck data, a separation in the focus direction of $1 \mathrm{~mm}$ was retrieved in the average location of LFI horns with respect to that of the HFI horns. Subsequent investigation concluded that in the geometry used for the RFFM, the LFI horns had been misplaced by this amount with respect to their actual as-built location. This was an unexpected and remarkable confirmation of the ability of the method to recover in-flight alignment parameters to sub-millimeter accuracy.
Table 5. Total power in radial bins for Model 2 and detector 217-1a.

\begin{tabular}{ccc}
\hline \hline Radial bin & Grating lobes & Power $(\%)$ \\
\hline $0.38-0.55 \ldots \ldots$ & Sinusoidal (SR) & 0.046 \\
$0.70-1.0 \ldots \ldots$ & S52 & 0.024 \\
$1.0-1.3 \ldots \ldots$ & $\ldots$ & 0.007 \\
$1.3-1.9 \ldots \ldots$ & M52 + S30 & 0.013 \\
\hline
\end{tabular}

After the initial tests on simulations, the method was adapted to use the very irregularly sampled Planck data (Borries et al. AMTA 2011). More specifically, a Kriging filter was included to bin and resample the measured samples onto a regular grid, exploiting physical priors on beam and noise properties. The method has also been used to recover grating lobes (Nielsen et al. 2011), but for this purpose the less automated Sect. 2 analysis is preferred.

The geometrical parameter space ( $x$ in Eq. (3)) that is explored includes (see Fig. 14):

- translation of the focal plane in three directions, rotation around the $z_{\mathrm{RDP}}$ axis, and a tilt of the $x, y$ plane around the $y$-axis;

- translation of each reflector in three directions and rotation around their respective $z$ axes;

- surface distortions of each reflector, modelled as Zernike polynomials up to third order;

- displacements of individual horns in $x_{\mathrm{RDP}}$ and $y_{\mathrm{RDP}}$.

The range of values explored for each parameter is determined from the very detailed thermoelastic model of the telescope system that was established prior to launch (Tauber et al. 2010, see Table 7). This model was used in a Monte Carlo analysis of a wide range of mechanical load cases; the ranges allowed in the optimization process were set to $\pm 1 \sigma$ values extracted from the Monte Carlo analysis. The rotation parameters were not constrained to a specific range, but the resulting deviations remained well within expectations. The allowed ranges for motion of individual horns with respect to their mounting plates (focal plane) 
J. A. Tauber et al.: The Planck telescope
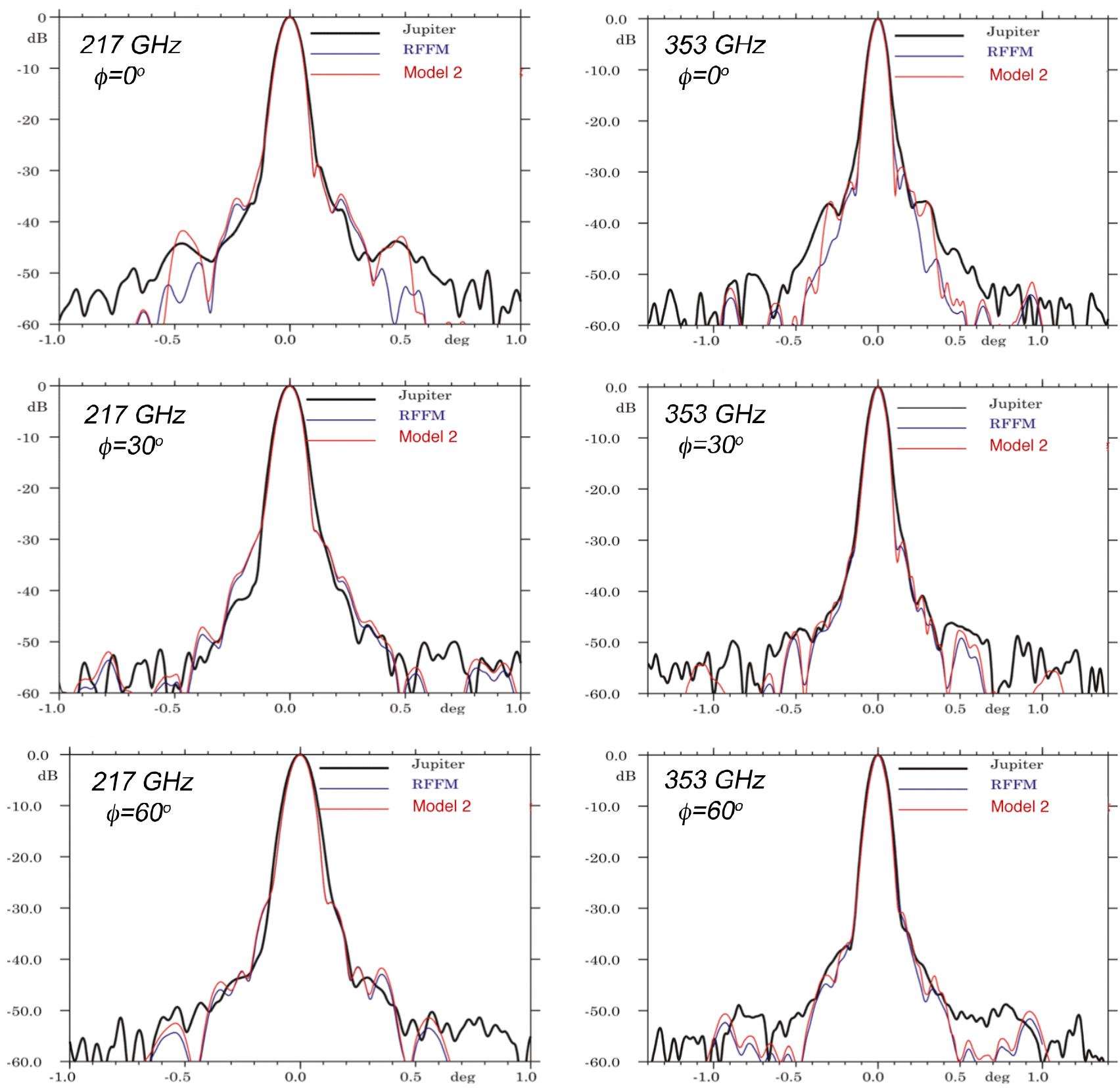

Fig. 12. Three radial cuts, at $\phi=0^{\circ}, 30^{\circ}$, and $60^{\circ}$, from the cross-scan directions. The curves show the measured pattern (black), the RFFM (blue), and Model 2 (red). $217 \mathrm{GHz}$ is shown on the left and $353 \mathrm{GHz}$ on the right.
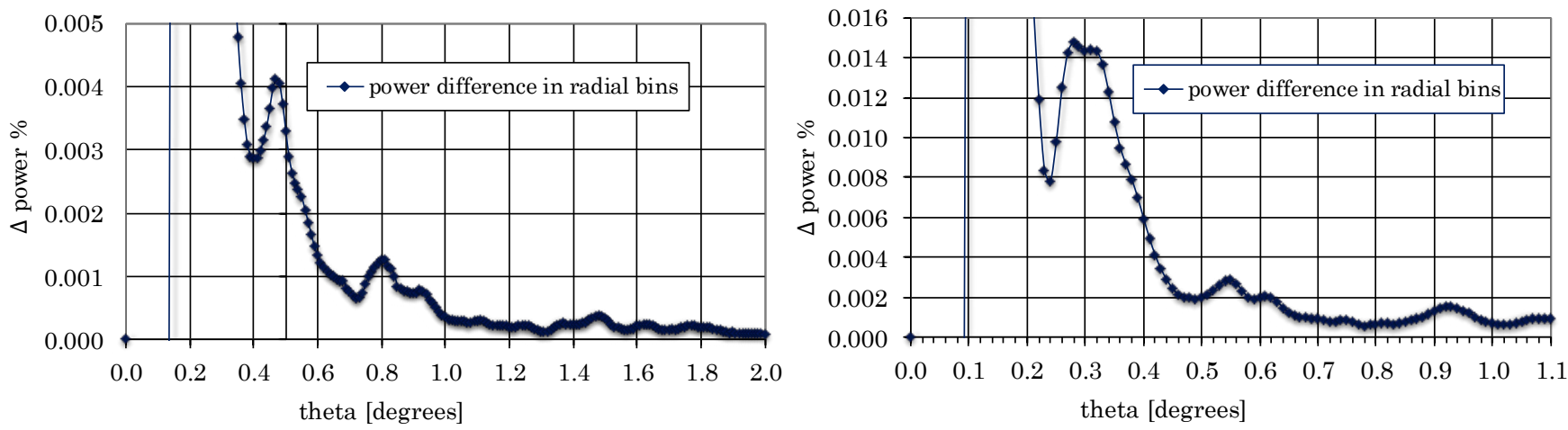

Fig. 13. Difference in measured and modelled power for two individual detectors (217-1a, left; and 353-1a, right), integrated in 0.01 radial bins. The angular regions of influence of each type of lobe can be seen in Tables 5 and 4 . 
Table 6. Total power in radial bins for Model 2 and individual lobes, at 217 and $353 \mathrm{GHz}$.

\begin{tabular}{lccccc}
\hline \hline & \multicolumn{2}{c}{$217 \mathrm{GHz}$} & & \multicolumn{2}{c}{$353 \mathrm{GHz}$} \\
\cline { 2 - 3 } \cline { 5 - 6 } $\begin{array}{l}\text { Typical power } \\
\text { Lobe Type }\end{array}$ & $\begin{array}{c}\text { All lobes } \\
\text { of this type }(\%)\end{array}$ & & $\begin{array}{c}\text { Typical power } \\
\text { in one lobe }(\%)\end{array}$ & $\begin{array}{c}\text { All lobes } \\
\text { of this type }(\%)\end{array}$ \\
\hline Sinus S90..... & 0.0099 & 0.04 & & 0.0017 & 0.07 \\
M52........ & 0.0012 & 0.007 & & 0.0035 & 0.02 \\
S52....... & 0.0041 & 0.025 & & 0.0053 & 0.03 \\
\hline
\end{tabular}

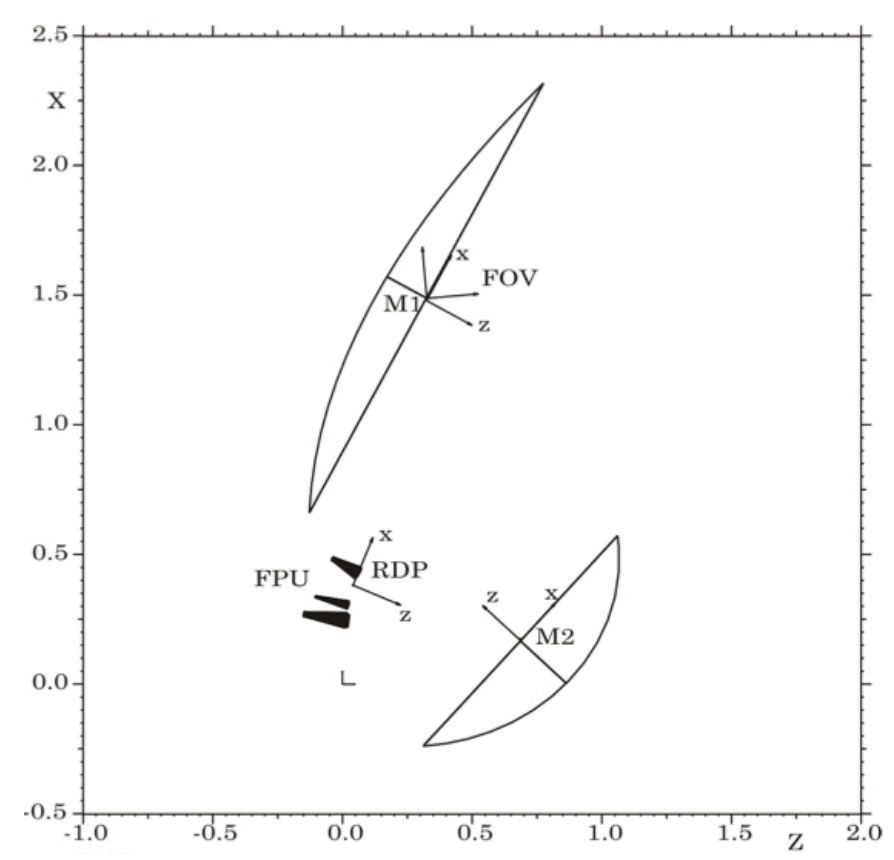

Fig. 14. Coordinate systems used in GRASP. RDP is the fiducial reference point, located in the RFFM focal plane. All other systems are referred to this point.

were determined by a combination of design tolerances and the uncertainty of in-situ measurement after their integration into the focal plane.

The parameter space contains many degeneracies and it would be impossible to explore it in a completely nondeterministic manner. Therefore a sequence of optimization runs was established that is physically motivated and gave good results on simulations. This sequence is listed below.

1. An initial model is selected, usually the RFFM (corrected for the originally misplaced LFI horns, as noted above).

2. The displacement of all beam centroids with respect to those of the initial model is determined (note that centroids are determined by fitting the full measured pattern to the model). The average deviation is removed from all measured beams under the assumption that it corresponds to an overall rotation of the optical system with respect to the absolute coordinate system determined in flight.

3. A first optimization is carried out, allowing only the alignment parameters (focal plane and reflector translations and rotations) to vary.

4. The locations of individual horns within the focal plane are now allowed to move to fit the new footprint. If any horns are found to require more than the allowed translation range ( $2 \sigma$, see Table 7$)$, they are excluded from the subsequent optimization.
5. Using the retrieved alignment parameters and new horn locations, a second optimization is carried out, allowing the reflector surface deformation parameters to vary.

6. The locations of individual horns within the focal plane are again allowed to move to fit the new footprint. If any horns are found to require more than the allowed translation range, they are excluded from the subsequent iteration.

7. the alignment parameters are now re-optimized, and individual horns translated to fit the footprint, to obtain the final configuration

8. The performance of the final configuration (including those horns that did not participate in the optimization) is evaluated.

This sequence of runs (which we refer to as a "retrieval") was first applied to a set of LFI beams, then to a joint set of LFI and HFI beams.

\subsection{Frequency dependence}

The prediction of modelled beams is made by GRASP at single frequencies within each detector's band. However, all Planck detectors have wideband response to radiation and the shape of the bandpass is far from flat (Planck Collaboration II 2014; Planck Collaboration IX 2014). Furthermore, the blackbody source spectrum changes across the maps made from planet observations, from a planet-like to a CMB-like temperature. The impact of not including these effects in the optimization has been modelled using realistic bandshapes for both Marslike and CMB-like spectra. In these simulations, the RFFM beam pattern was calculated using GRASP at five individual frequencies across each band. The five patterns were then convolved with the bandpass response and source spectrum (binned appropriately). The resulting "wideband" patterns were compared to the monochromatic ones. Using a metric similar to the variance used to assess deviations between modelled and measured beams (Eq. (3)), we conclude that the differences between monochromatic and wideband beams are not significant for our analysis. For illustration, we show some patterns in Fig. 15, which indicate that significant changes start to appear at the $-30 \mathrm{~dB}$ contour, whereas this analysis only uses patterns down to $-15 \mathrm{~dB}$ (LFI) and $-20 \mathrm{~dB}$ (HFI). We conclude from these tests that down to the typical signal levels used in the table, we can safely use monochromatic beams to fit those measured on Jupiter.

\subsection{The input data}

\subsubsection{LFI data}

The LFI data that we use includes seven passes of Jupiter for all 22 detectors, processed by a pipeline that in terms of calibration, cleaning, and pointing, corresponds to the standard processing 
Table 7. Ranges of mechanical motions allowed.

\begin{tabular}{|c|c|c|}
\hline Element & Parameter & Range $(\mathrm{mm})$ \\
\hline LFI focal plane......... & Translation & \pm 0.7 \\
\hline HFI-LFI focal plane...... & $z$ Translation & \pm 0.1 \\
\hline Primary.............. & Translation & \pm 0.3 \\
\hline Secondary............ & Translation & \pm 0.6 \\
\hline Feedhorn.............. & Translation & \pm 0.1 \\
\hline
\end{tabular}

Table 8. Results of the LFI-only retrieval.

\begin{tabular}{|c|c|}
\hline Element & Change \\
\hline \multicolumn{2}{|l|}{ LFI FPU } \\
\hline Cross-scan offset... & +1.82 \\
\hline In-scan offset...... & -1.55 \\
\hline$x$ translation...... & $-0.06 \mathrm{~mm}$ \\
\hline$y$ translation...... & $-0.01 \mathrm{~mm}$ \\
\hline$z$ translation...... & $+0.05 \mathrm{~mm}$ \\
\hline Rotation.......... & $-4 ! 1$ \\
\hline$p-p(x)^{a} \ldots \ldots \ldots$ & $0.54 \mathrm{~mm}$ \\
\hline$p-p(y)^{b} \ldots \ldots \ldots$ & $0.44 \mathrm{~mm}$ \\
\hline \multicolumn{2}{|l|}{ Primary } \\
\hline$z$ translation....... & $0.0 \mathrm{~mm}$ \\
\hline Rotation......... & $0^{\circ}$ \\
\hline \multicolumn{2}{|l|}{ Zernike $\operatorname{mode}(m, n)^{b}$} \\
\hline$(0,2) \ldots \ldots \ldots$ & $+0.13 \mathrm{~mm}, 0^{\circ}$ \\
\hline$(1,1) \ldots \ldots \ldots$ & $+1.09 \mathrm{~mm},-55^{\circ}$ \\
\hline$(2,2) \ldots \ldots \ldots$ & $+0.05 \mathrm{~mm},-45^{\circ}$ \\
\hline$(1,3) \ldots \ldots \ldots$ & $+0.06 \mathrm{~mm},-1^{\circ}$ \\
\hline$(3,3) \ldots \ldots \ldots$ & $+0.02 \mathrm{~mm},-52^{\circ}$ \\
\hline \multicolumn{2}{|l|}{ Secondary } \\
\hline$z$ translation...... & $0.0 \mathrm{~mm}$ \\
\hline Rotation........... & $0^{\circ}$ \\
\hline \multicolumn{2}{|l|}{ Zernike mode $(m, n)^{b}$} \\
\hline$(0,2) \ldots \ldots \ldots$ & $+0.03 \mathrm{~mm}, 0^{\circ}$ \\
\hline$(1,1) \ldots \ldots \ldots$ & $+1.67 \mathrm{~mm},-28^{\circ}$ \\
\hline$(2,2) \ldots \ldots \ldots$ & $+0.05 \mathrm{~mm},-54^{\circ}$ \\
\hline$(1,3) \ldots \ldots \ldots$ & $+0.32 \mathrm{~mm},-6^{\circ}$ \\
\hline$(3,3) \ldots \ldots \ldots$ & $+0.06 \mathrm{~mm},+54^{\circ}$ \\
\hline
\end{tabular}

Notes. ${ }^{(a)} p-p(x)=$ largest remaining displacement differences in the $x$-direction between retrieved model and measured detector locations in the focal plane. ${ }^{(b)}$ Zernike mode $(0,2)=$ defocus; $(1,1)=$ tilt; $(2,2)=$ ellipticity.

used for the Planck 2015 data release (Planck Collaboration II 2016). All data samples around Jupiter were put onto a $(u, v)^{8}$ spherical grid centred on the line-of-sight system.

The LFI planet data as delivered are "smeared," i.e., they contain the effect of sampling time as the beam scans the sky, which in effect corresponds to a boxcar filtering in the time (or angular) domain. In the parlance of the LFI and HFI DPCs, the map of Jupiter made from these samples corresponds to the "scanning beam," which is slightly wider in the scan direction than the "optical beam". This effect is deconvolved from the delivered data before any fitting is made, by means of a Wiener filter in the Fourier domain of the time samples.

\footnotetext{
8 Here $u=\sin ($ cross - scan $) \sim$ cross - scan and $v=\sin ($ in - scan $) \times$ $\cos ($ cross - scan $) \sim$ in-scan.
}

All LFI detectors (including two polarizations per horn) were initially used, though during the optimization process the data for LFI-20 and $21(70 \mathrm{GHz})$ were discarded, since their individual centroid locations were too far from the predicted ones. The method was applied to all individual samples for each detector, i.e., there was no a priori stacking of the individual Jupiter pass data. Only data samples with signal amplitude above $-15 \mathrm{~dB}$ from peak was used in the optimization; for comparison, the noise floor of a stack of all Jupiter passes for individual detectors corresponds to around $-29,-27$, and $-20 \mathrm{~dB}$ from peak at 30, 44, and $70 \mathrm{GHz}$, respectively. This conservative threshold ensures that all samples have adequate $\mathrm{S} / \mathrm{N}$ and no significant effects from wideband response (see Sect. 3.2) are expected.

\subsubsection{HFI data}

The HFI data are affected not only by the angular response of each detector ${ }^{9}$, but also by the complex time response of each bolometer. Planet maps cannot therefore be directly compared to optical models. For scientific analysis of HFI data, "scanning beams" are produced, using planet maps from which the time response has been deconvolved (Planck Collaboration VII 2016; Roudier et al. 2015). In the deconvolution process a lowpass filter is applied to prevent division by zero around the nulls of the time response's Fourier transform (which occur at the odd harmonics of the signal deconvolution frequency). The lowpass filtering distorts the scanning beam significantly. In order to avoid this distortion, a B-spline-based beam model is convolved with the time response function ${ }^{10}$ and fit to each detector's planet maps to produce "forward-sense beams" ("fsbeams" for short), which are considered the best approximation to the optical response (but not optimal for scientific analysis).

The relative location of all the HFI beams is derived from the scanning beams. However, the absolute directions of the beam centroids are degenerate with a timing delay between the pointing sensors (star tracker) and the bolometer acquisition electronics. To break this degeneracy, one set of observations of Mars was made with the satellite spinning at $1.4 \mathrm{rpm}$ instead of the usual $1 \mathrm{rpm}$. Comparison of maps with different scanning speeds yielded the offset, which is about $4^{\prime}$ in the scanning direction and 0.1 in the cross-scan direction. The offset is not the same for each detector, and the rms variation is of order 0'1. Due to restrictions in satellite pointing, only a subset of the focal plane (covering the 100, 143, and $217 \mathrm{GHz}$ detectors) was able to observe Mars in the spun-up configuration; for all other detectors, the average of the individual estimated offsets was applied. Note, however, that in the retrieval described in this paper, the scanning-beam-based directions only are used as the initial determination of the focal plane coordinate system - thereafter all beam centroid directions are determined by fitting model beams to the measured ones.

For each of the two main Planck data releases so far (in 2013 and 2015), a set of fsbeams was produced specifically for the exercise described here, in both cases based on maps of Saturn and Jupiter. There were significant differences in the way the fsbeams were produced in 2013 and 2015 (Planck Collaboration VII 2016; Planck Collaboration VI 2014), including: number of planet maps used; size of the fields recovered; and, most importantly, different time transfer func-

9 These consist of back-to-back horns, filters, and a bolometer in a cavity (or two in the case of polarization-sensitive bolometers).

${ }^{10}$ This is itself determined from a combination of ground and in-flight data (Planck Collaboration VII 2016). 

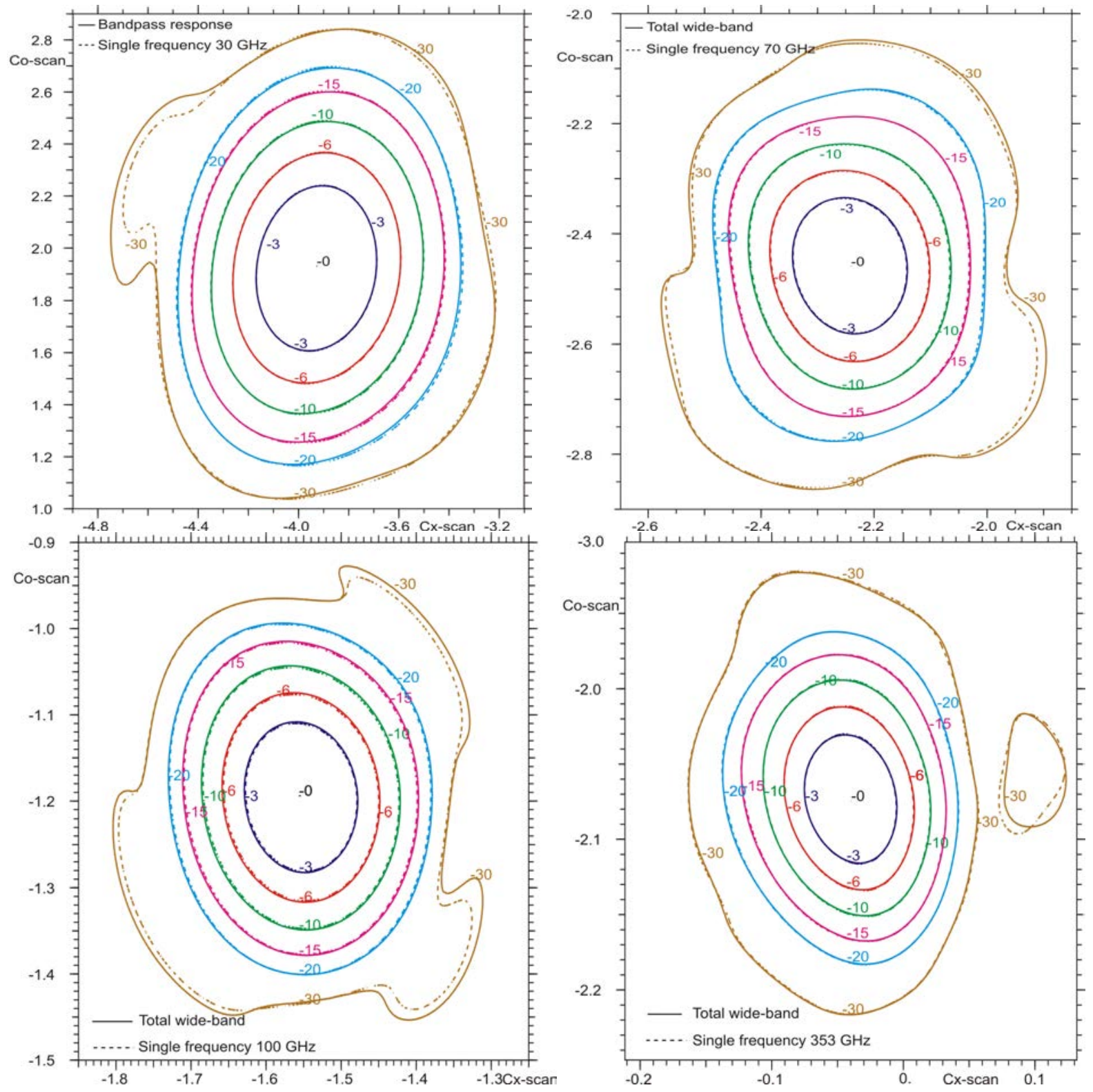

Fig. 15. Difference in predicted patterns between monochromatic and wideband models. The four detectors shown are $27 \mathrm{~S}(30 \mathrm{GHz}), 18 \mathrm{~S}(70 \mathrm{GHz})$, $1 \mathrm{a}(100 \mathrm{GHz})$, and $1(353 \mathrm{GHz})$. The bandpass shape is realistic and the source spectrum assumed here is CMB (but a similar result is obtained when the source spectrum assumed is that of Mars). In this and all subsequent beam contour plots, the axis units are in degrees.

tions. In 2013, the central parts of the "scanning" beams used in the scientific analysis were based on Mars maps instead.

Some of the 2015 fsbeams were found to contain small-scale ripples that could not be optical in origin. These features are interpreted as due to non-removed glitches that excite high frequencies along the scanning direction. Eight fsbeams were discarded for this reason, and a further 10 fsbeams were not used because the quality of the time-transfer determination was considered "medium" 11 . It was also noted that several pairs of PSB fsbeams show differences that are not reproduced by models, both in the peak direction and in the beam shape, typically at the level of $15-20 \mathrm{~dB}$ from peak contour and in the scan direction; these fsbeams were also not used. All of these factors imply that the fsbeams are a poorer representation of the optical response of each detector than initially assumed.

11 The labels of the bolometers that were kept in the retrieval were: 100-1a, 100-4a, 143-5, 143-7, 217-1, 217-3, 353-1, and 353-8.
In total 11 (2015) fsbeams remained in the range 100$353 \mathrm{GHz}$ for this exercise (the multi-moded nature of the 545 and $857 \mathrm{GHz}$ detectors precluded their use for this purpose). All data samples with signal amplitude above $-20 \mathrm{~dB}$ from peak were used in the retrieval.

\subsection{Results}

\subsubsection{LFI-only retrieval}

For the retrieval of the geometry using only LFI beams, all detectors were initially given the same weight. In all retrieval iterations, it was noted that the retrieved $z$ displacements of the PR and SR always end up at the same end of their allowed range ( -0.3 and $-0.6 \mathrm{~mm}$, respectively). The effect of these two parameters (or rather of their difference) is degenerate with the $z$ displacement of the focal-plane unit (FPU) and with the $(0$, 2) Zernike mode of the reflectors (defocus). Indeed, increasing 
the allowed displacement range for the two reflectors leads to pegging at the end of their new ranges, which is being compensated by corresponding increases in the degenerate parameters and hardly any change in all other parameters, such that the final performance is only improved in a very insignificant way (a similar but more pronounced behaviour is observed when performing retrievals with HFI fsbeams). Therefore we have preferred to fix the $z$ displacements of the PR and SR to their nominal values, avoiding unrealistic PR and SR displacements.

The geometrical parameters retrieved using all LFI data are summarized in Table 8. The total variance (Eq. (2)) of the final configuration was reduced from $0.2 \mathrm{~dB}$ (for the RFFM) to $0.089 \mathrm{~dB}$. The tables show physically reasonable parameters (see Table 7). The retrieved Zernike mode $(3,3)$ distortions are aligned with the triangles defined by the ISMs, which means that they are physically motivated. With the exception of two horns (LFI20 and LFI21), the deviations of individual horns with respect to their nominal locations are near to or within $1 \sigma$ of expectations, and are randomly distributed in direction (indicating that no systematic misalignment effects remain). We have no clear explanation for the relatively large residual misalignment of horns LFI 20 and LFI21; although their location next to each other around the mid-plane of the FPU supports a mechanical origin, the pre-flight front-end module analysis did not show any anomalous behaviour in this particular location.

The new LFI configuration yields excellent fits between measured and modelled beams, especially when compared to the starting point. We illustrate this in Fig. 16, with two individual detectors at $70 \mathrm{GHz}$ for which the fit was particularly good (20S) and particularly poor (18S) from the point of view of the final variance ( 0.03 and 0.08 , respectively). The contours of the model and measured patterns are almost indistinguishable for both detectors down to $-20 \mathrm{~dB}$ from peak. A more discriminating measure can be appreciated with the power differences integrated in radial bins (shown also in Fig. 16); here we see that there remain differences of order $0.2-0.4 \%$ in our ability to predict the beams with the models.

We classify fits as "good" or "bad" according to the variance. However, Fig. 16 illustrates the fact that the contour plots by themselves do not clearly reflect the goodness of fit - indeed the differences between the selected good and the poor fit examples cannot easily be appreciated by eye; the radial power profiles are easier to interpret but also do not reflect the entire picture. We recommend that to assess the quality of a model against the data, not only the variance, but also the radial power plots, as well as the ensemble of characteristics listed in Table 8, should be examined together.

\subsubsection{Joint $\mathrm{LFI}+\mathrm{HFI}$ retrieval}

Initially we tried HFI-only retrievals, but they yielded mechanically unrealistic configurations (with misalignments of the FPU and PR/SR distortions of order $\mathrm{mm}$ ). This was not the case with much earlier retrievals, which were based on "naively"-derived beams based on single-season observations of Mars, and yielded satisfactory HFI-only fits (especially considering the paucity of the data included). This result reinforces the conclusion that the process producing fsbeams generates features that are not optical ones or suppresses optical ones, even in those cases that have not been discarded for obvious defects (see Sect. 3.3.1). As an example, Fig. 17 shows that Mars-based beams are more elongated than the fsbeams, and it is this type of low-order distortion that could cause the first steps of the retrieval process (Sect. 3.1) to fail.
In any case, since the HFI is mechanically attached to the LFI, it makes sense to use the best "LFI-only" retrieval as a starting point for retrievals using HFI fsbeams. In effect this makes such retrievals joint LFI+HFI ones. The question then becomes how to best combine LFI and HFI beams in the retrieval process so as not to let it be misled by the suspicious fsbeams ${ }^{12}$. Our solution has been to tune the methodological steps described in Sect. 3.1, giving more weight to the LFI for the initial steps and adding HFI-based information in the later iterations. Having tried several different approaches, the best-performing joint configuration using 2015 fsbeams resulted from: fixing the LFI and telescope to the best LFI-only retrieval (Sect. 3.3.1) and retrieving HFI-specific alignment parameters (location of FPU and individual detectors); then fixing both LFI and HFI to retrieve new PR/SR distortion parameters; iterating on the LFI and HFI FPU locations, with a constraint on relative distance; and finally iterating on all individual detector parameters. This process balances the relative influences of LFI and HFI.

The 2015 retrieved configuration improves the performance of the HFI model over that of the RFFM: the variance (Eq. 2) is reduced from 0.8 to 0.42 . However, the performance of the LFI model (variance about 0.2 ) is degraded with respect to the LFI-only retrieval (Sect. 3.3.1) and is in fact not better than that of the RFFM. Furthermore, the dispersion of the remaining detector displacements is quite large $(0.5 \mathrm{~mm})$, and the FPU is displaced in the $z$-direction by $0.5 \mathrm{~mm}$. Particularly the former makes this a poor solution. The fact that the addition of the HFI caused the LFI-only solution to be significantly degraded reinforces the suspicion that the 2015 fsbeams contain non-optical features that are affecting the solution.

Therefore we carried out a similar exercise replacing the HFI 2015 fsbeams with those produced in 2013, which, as pointed out in Sect. 3.3.2, are generated using the same basic algorithm, but with significantly different inputs (notably, using Mars data for the inner parts of the beams). The results of the joint retrieval using 2013 fsbeams are summarized in Table 9, and the residual displacements and variances for individual detectors are shown in Tables 10 and 11. The residual displacements should be compared to the mechanical tolerances (Table 7). The residual variances give an indication of the relative goodness of fit of the model patterns to the measurements ${ }^{13}$.

The joint retrieval based on the 2013 fsbeams (Table 9) performs significantly better than that using 2015 fsbeams.

- The performance of the LFI model (variance about 0.13 ) is significantly better than that of the RFFM $(0.2 \mathrm{~dB})$. Note that the LFI FPU displacements remain fixed to the results obtained for the LFI-only retrieval.

- The performance of the HFI model (variance about 0.38 ) is better than that of the 2015 model (variance about 0.42 ) and that of the RFFM (variance about 0.8 ).

- The locations of the FPU and the reflectors are close to their nominal locations.

- The amplitudes of the Zernike distortions of PR and SR are within tolerances, and their directions are aligned with the triangle defined by the reflector ISMs.

- The remaining displacements of all LFI detectors are within or near their $1 \sigma$ expectations, with the exception of LFI20 and LFI21, whose displacements are between 3 and $4 \sigma$. They are also randomly distributed in direction (see Fig. 18).

\footnotetext{
12 Initially we planned to weight individual beams according to their "quality," but we could not find a way to do this objectively.

${ }^{13}$ LFI and HFI residual variances should not be directly compared to
} each other, since their normalizations are quite different. 

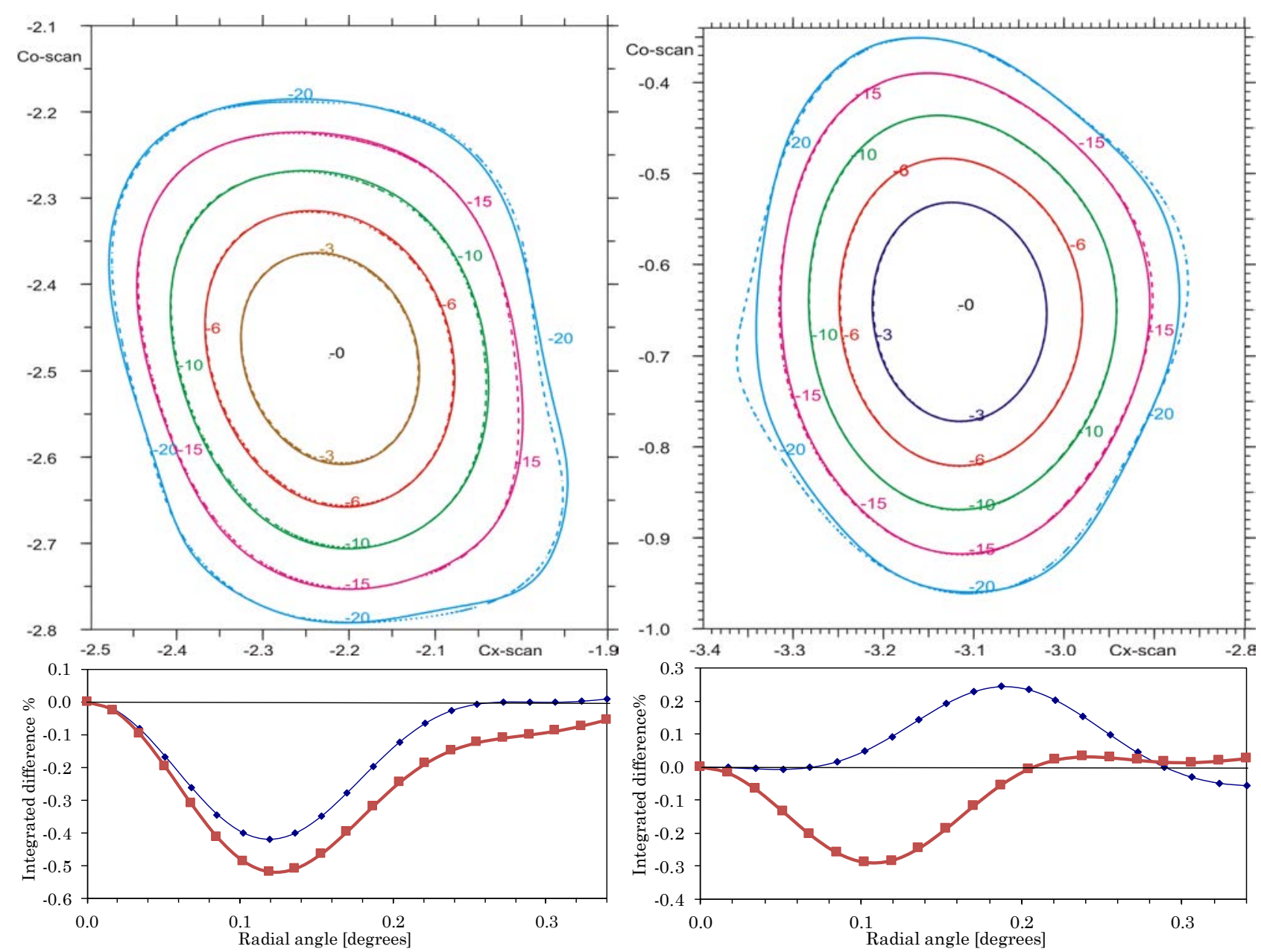

Fig. 16. Top panels: comparison of measured patterns (full line) to the corresponding LFI-only best-fit retrieved patterns (dashed line), for two individual detectors at $70 \mathrm{GHz}$ : on the left is an example of a poor fit (high variance $\delta$ ), $18 \mathrm{~S}$; and on the right a good fit (low variance), 20S. Bottom panels: difference in power between the above patterns, integrated up to a given radial angle, as a function of that angle; these plots are useful to identify where the major differences occur. We also show the equivalent curves (in red) for the RFFM pattern.
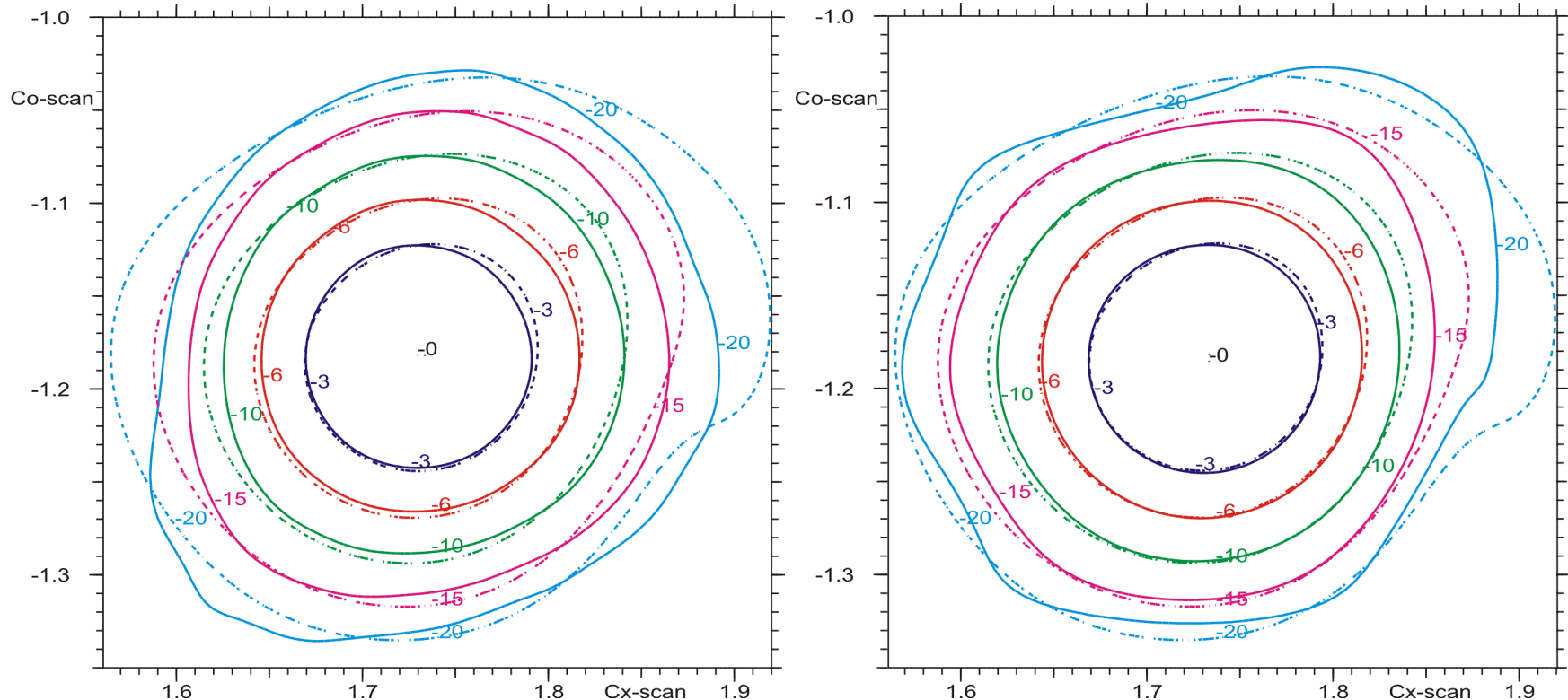

Fig. 17. Left panel: comparison of fsbeam of detector 143-5 (full line) to the retrieved model (dashed line). Right panel: comparison of Mars-based beam of detector 143-5 (full line) to the retrieved model (dashed line). The Mars-based beam shows extended modelled shoulders that are not present in fsbeam, but are present in the model beam. The inference is that the use of fsbeam introduces non-optical features in the beam. 
Table 9. Results of the LFI+HFI retrieval, based on 2013 fsbeams.

\begin{tabular}{|c|c|}
\hline Element & Change \\
\hline \multicolumn{2}{|l|}{ LFI FPU } \\
\hline Cross-scan offset... & +0.14 \\
\hline In-scan offset...... & +0 ' 19 \\
\hline$x$ translation...... & $-0.06 \mathrm{~mm}$ \\
\hline$y$ translation...... & $-0.01 \mathrm{~mm}$ \\
\hline$z$ translation...... & $+0.05 \mathrm{~mm}$ \\
\hline Rotation......... & $-4 ! 1$ \\
\hline$p-p(x) \ldots \ldots \ldots$ & $0.35 \mathrm{~mm}$ \\
\hline$p-p(y) \ldots \ldots \ldots$ & $0.27 \mathrm{~mm}$ \\
\hline \multicolumn{2}{|l|}{ HFI FPU } \\
\hline Cross-scan offset... & -0.51 \\
\hline In-scan offset...... & -0.12 \\
\hline$x$ translation...... & $+0.27 \mathrm{~mm}$ \\
\hline$y$ translation...... & $-0.11 \mathrm{~mm}$ \\
\hline$z$ translation...... & $+0.26 \mathrm{~mm}$ \\
\hline Rotation......... & +0.9 \\
\hline$p-p(x) \ldots \ldots$. & $0.20 \mathrm{~mm}$ \\
\hline$p-p(y) \ldots \ldots \ldots$ & $0.26 \mathrm{~mm}$ \\
\hline \multicolumn{2}{|l|}{ Primary } \\
\hline$z$ translation...... & $0.0 \mathrm{~mm}$ \\
\hline Rotation......... & $0^{\circ}$ \\
\hline \multicolumn{2}{|l|}{ Zernike mode $(m, n)$} \\
\hline$(0,2) \ldots \ldots \ldots$ & $+0.08 \mathrm{~mm}, 0^{\circ}$ \\
\hline$(1,1) \ldots \ldots \ldots$ & $+0.99 \mathrm{~mm},+68^{\circ}$ \\
\hline$(2,2) \ldots \ldots \ldots$ & $+0.13 \mathrm{~mm},+79^{\circ}$ \\
\hline$(3,3) \ldots \ldots \ldots$ & $+0.09 \mathrm{~mm},-6^{\circ}$ \\
\hline$(1,3) \ldots \ldots \ldots$ & $+0.06 \mathrm{~mm},-58^{\circ}$ \\
\hline \multicolumn{2}{|l|}{ Secondary } \\
\hline$z$ translation...... & $0.0 \mathrm{~mm}$ \\
\hline Rotation......... & $0^{\circ}$ \\
\hline Zernike mode $(m, n)$ & \\
\hline$(0,2) \ldots \ldots \ldots$ & $! 0.0 * \mathrm{~mm}, 0^{\circ}$ \\
\hline$(1,1) \ldots \ldots \ldots$ & $+1.37 \mathrm{~mm},-35^{\circ}$ \\
\hline$(2,2) \ldots \ldots \ldots$ & $+0.05 \mathrm{~mm},-69^{\circ}$ \\
\hline$(1,3) \ldots \ldots \ldots$ & $+0.29 \mathrm{~mm},-7^{\circ}$ \\
\hline$(3,3) \ldots \ldots \ldots$ & $+0.03 \mathrm{~mm},+4^{\circ}$ \\
\hline
\end{tabular}

Notes. ${ }^{(a)} p-p(x)=$ largest remaining displacement differences in the $x$-direction between retrieved model and measured detector locations in the focal plane. ${ }^{(b)}$ Zernike mode $(0,2)=$ defocus; $(1,1)=$ tilt; $(2,2)=$ ellipticity.

- The remaining displacements of most HFI detectors are well within their $1 \sigma$ expectation, with a few of them exceeding $1 \sigma$ slightly. However, the directions of these residuals are not randomly distributed, and indicate that systematic effects remain present (see Fig. 18).

We note that the HFI FPU is translated in the $x$-direction by approximately $0.3 \mathrm{~mm}$ with respect to the LFI FPU. This value is large (about $3 \sigma$ ), but is consistently retrieved in all iterations. It corresponds to an average pointing displacement of $0.9^{\prime}$ in the cross-scan direction. It is possible that this feature is a consequence of focal-plane reconstruction based on pointing, rather than on a mechanical displacement.

The model retrieved from the 2015 LFI beams and the 2013 HFI fsbeams is the best joint geometrical model that we can produce at this time. Nonetheless, there are good reasons to remain suspicious of the "optical" quality of the HFI fsbeams; it seems likely that the forward-sense algorithm introduces nonoptical features, or suppresses real ones, and these distortions are affecting the retrieval. Indeed, as mentioned earlier, the HFI model beam shapes are - in some cases at least - closer to beams derived naively from Mars data only than to the fsbeams (see one example in Fig. 17). Even if the 2013 fsbeams appear to be less prone to such effects than the 2015 beams, their presence cannot be discarded. In view of this, and considering that the LFI variance of the joint retrieval is slightly degraded from that of the LFI-only retrieval (variance 0.089), it is legitimate to ask whether the LFI-only retrieval is a better representation of the telescope and LFI FPU than the joint one. However, we note that some results of the joint solution are improved over the LFI-only retrieval: in particular, the residual detector displacements are significantly smaller and closer to expectations. Overall, it is not straightforward to determine which of the two is the best solution for LFI. Considering that we have down-weighted the low-order information in the HFI beams (as most likely to be contaminated by time-response effects), the joint retrieval remains in our view the best compromise solution. We refer to this model as RFFM2, and we will make it publicly available via the Planck Legacy Archive ${ }^{14}$.

We illustrate the performance of the retrieved model with some examples of the patterns in Figs. 19 and 20. In these figures we include contour plots of measured and modelled beams, as well as the radially integrated power difference, for both the best-fit model and the RFFM. The peak power difference of the radially integrated power is reported in Tables 10 and 11. These differences are indicative of the quality of the fit to the data, ranging over $0.1-1.3 \%$ for LFI and over $0.3-2.5 \%$ for HFI. These are clearly much larger than the uncertainty levels required for cosmological analysis of the Planck data, which is of order $0.1 \%$.

\section{Emissivity}

The emissivity is an important characteristic of a telescope such as Planck, since it contributes directly to its noise budget, especially at the high end of its frequency range. The total emissivity of the Planck telescope was required to be below $0.6 \%$ at beginning of life (BOL), and was expected to increase over the mission lifetime due to dust contamination and degradation of the surface through UV radiation and micro-meteoroid impacts. The emissivity of sample surfaces similar to the Planck reflectors were measured prior to launch (Tauber et al. 2010) and were shown to have a strong dependence on: (a) the physical properties of the aluminium reflecting layer; (b) the protective (Plasil) coating; and (c) the temperature. Extrapolation to in-flight operating conditions indicated that the Planck reflectors should have emissivity levels at BOL lower than required: $<0.05 \%$ at $50 \mathrm{GHz}$ to $<0.15 \%$ at $350 \mathrm{GHz}$ (without including a potential contribution from dust deposition during launch).

The telescope emissivity was estimated by HFI in flight (Planck Collaboration II 2011) at BOL from the background power remaining after accounting for all other known sources, and yielded a level of $0.07 \%$ across the entire HFI frequency range, with large uncertainties $(>100 \%)$. In the final phases of the mission, before disposal of the satellite, LFI conducted a dedicated test that consisted of heating the primary and secondary reflectors by a few degrees, and measuring the power excess detected by the LFI radiometers. This measurement is described in detail in Cuttaia et al. (2019), and leads to an estimate of the emissivity in the range $0.04-0.06 \%$ between 30 and $70 \mathrm{GHz}$,

14 http://pla.esac.esa.int/pla 

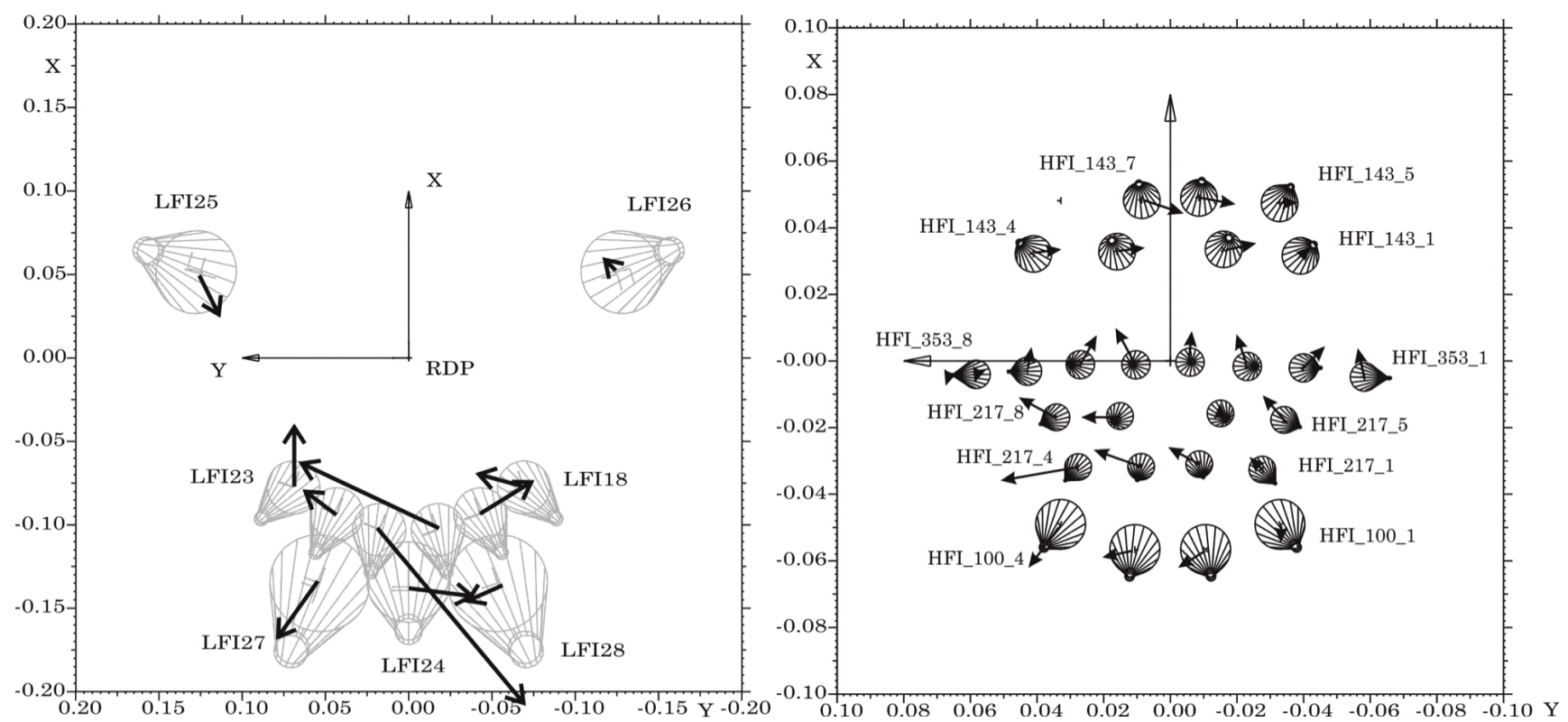

Fig. 18. Left panel: displacement residuals remaining on LFI detectors after removing the joint fit. Right panel: residuals remaining on HFI detectors after removing the joint fit. The axis units are $\mathrm{mm}$.
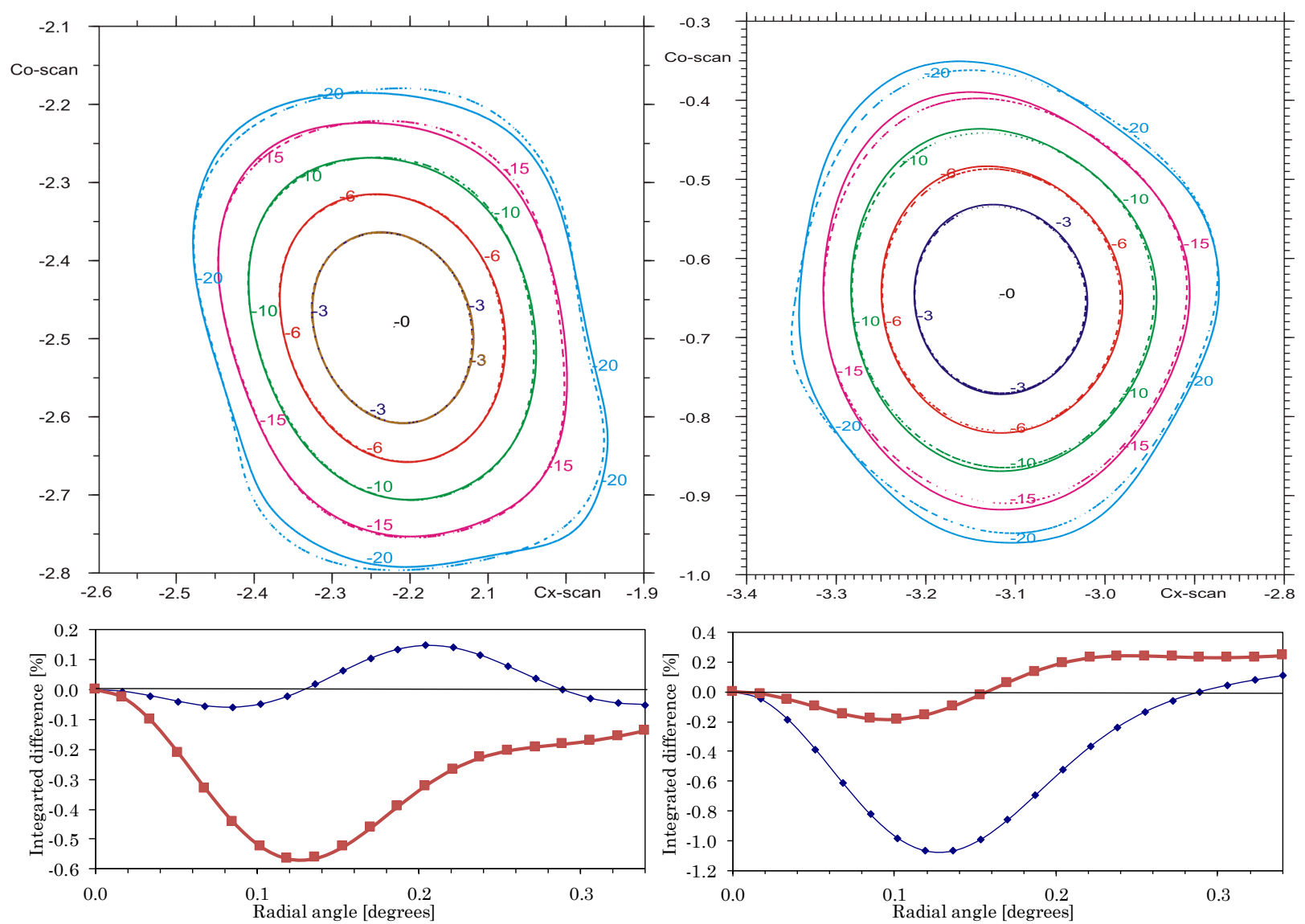

Fig. 19. Top panels: LFI measured beams (full line) for two individual detectors at $70 \mathrm{GHz}$ (left, 18S; right, 20S) compared with the corresponding joint best-fit retrieved patterns (dashed line); the left panel is representative of a good fit (high variance $\delta$ ), and the right one of a poor fit (low variance). These are the same detectors used to illustrate the LFI-only fit in Fig. 16. Bottom panels: difference in power between the above patterns, integrated up to a given radial angle, as a function of that angle; the difference between the measured patterns and the RFFM patterns (initial condition) is also shown (red curves). We note that Detector 20S is quite anomalous and was not used in the joint retrieval; indeed both the RFFM and the LFI-only retrieval models are better fits to the data than the joint retrieval. 

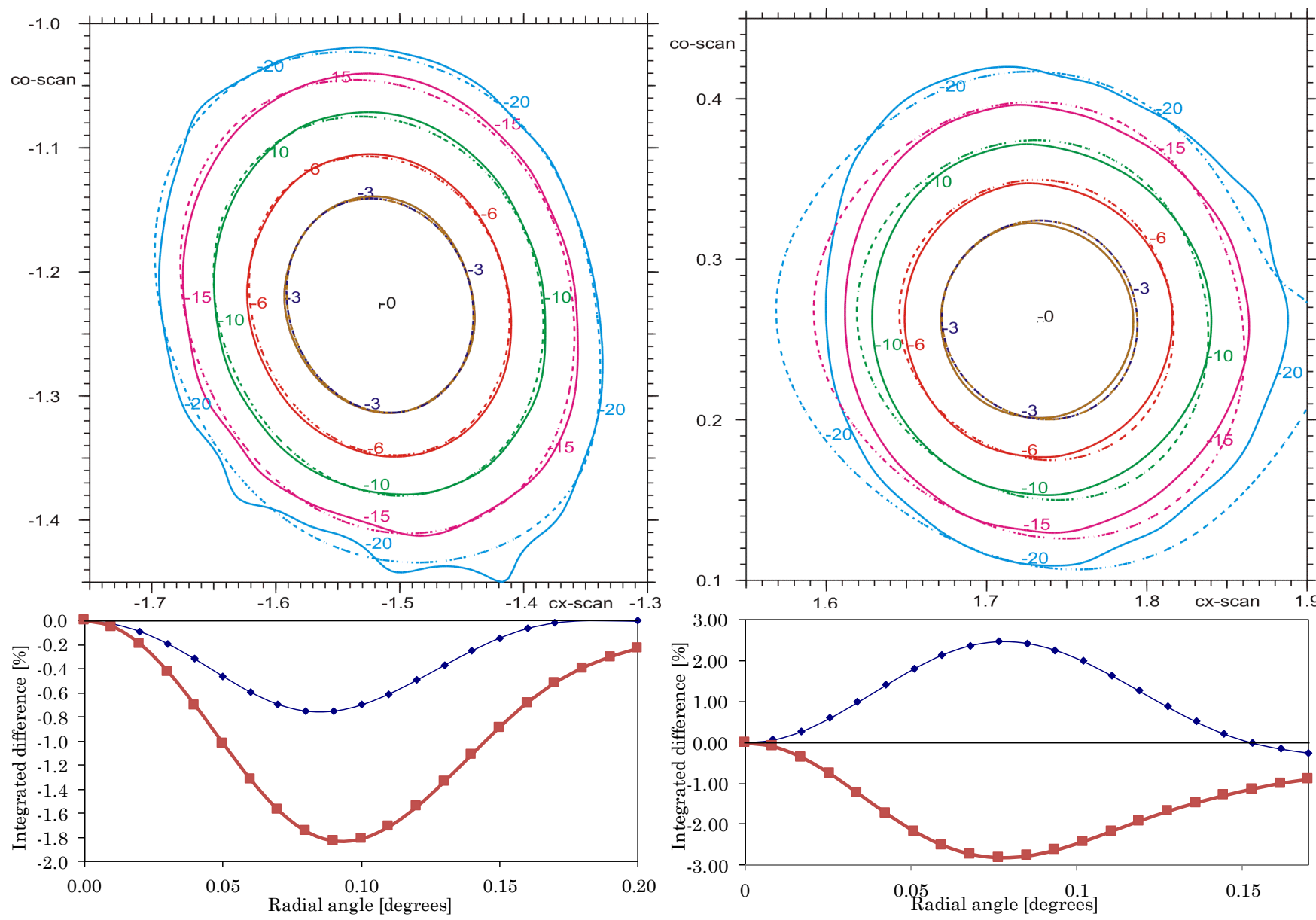

Fig. 20. Top panels: HFI fsbeams (full line) for two individual detectors (left, 100-1a; right, 147-7) compared with the corresponding joint best-fit retrieved patterns (dashed line); the left panel is representative of a good fit, and the right one of a poor fit. Bottom panels: difference in power between the above patterns, integrated up to a given radial angle, as a function of that angle; the difference between the measured patterns and the RFFM patterns (initial condition) is also shown (red curves).

Table 10. Residuals of RFFM2 for individual LFI detectors.

\begin{tabular}{ccccccc}
\hline \hline & \multirow{2}{*}{$\begin{array}{c}\text { Displacement }(\mathrm{mm}) \\
\text { Detector }\end{array}$} & $(\Delta x, \Delta y)$ & \multicolumn{2}{c}{ Variance $(\mathrm{dB})$} & & \multicolumn{2}{c}{ MPD $(\%)$} \\
\cline { 6 - 7 } & & S-pol & M-pol & & S-pol & M-pol \\
\hline $70-18 \ldots \ldots$ & $(0.03,0.11)$ & 0.08 & 0.08 & & 0.1 & 0.2 \\
$70-19 \ldots \ldots$ & $(0.08,-0.13)$ & 0.10 & 0.15 & & 0.5 & 1.0 \\
$70-20 \ldots \ldots$ & $(0.16,0.34)$ & 0.15 & 0.16 & & 1.1 & 1.3 \\
$70-21 \ldots \ldots$ & $(-0.43,-0.36)$ & 0.17 & 0.15 & & 1.3 & 1.1 \\
$70-22 \ldots \ldots$ & $(0.06,0.08)$ & 0.08 & 0.10 & & 0.3 & 0.3 \\
$70-23 \ldots \ldots$ & $(0.15,0.00)$ & 0.10 & 0.10 & & 0.6 & 0.5 \\
$44-24 \ldots \ldots$ & $(-0.20,-0.16)$ & 0.10 & 0.07 & & 0.7 & 0.1 \\
$44-25 \ldots \ldots$ & $(-0.10,-0.05)$ & 0.13 & 0.17 & & 0.5 & 0.5 \\
$44-26 \ldots \ldots$ & $(0.03,0.03)$ & 0.10 & 0.19 & & 0.2 & 0.2 \\
$30-27 \ldots \ldots$ & $(-0.14,0.10)$ & 0.13 & 0.10 & & 0.4 & 0.3 \\
$30-28 \ldots \ldots$ & $(-0.04,0.09)$ & 0.14 & 0.12 & & 0.4 & 0.3 \\
\hline
\end{tabular}

Notes. MPD is the radial maximum of the integrated power difference between the measured and modelled beams. S-Pol and M-Pol label each of the two orthogonally-polarized receiver in each LFI detector.

which is consistent with both the HFI BOL estimate and the onground measurements. On this basis, it is possible to say that there was no major degradation of the emissivity during the lifetime of the mission, but the large uncertainties do not allow us to make more detailed statements.

\section{Synthesis of the results}

\subsection{Small-scale reflector structure}

Fitting stacked Jupiter HFI maps to models of the physical structure of the Planck reflectors yields the following conclusions.

1. The pre-launch RFFM requires some modifications to the reflector surfaces to account for grating lobes:

- the measured SR deformations at small scales need to be amplified by a factor of 1.2 ;

- a constant dimpling of peak amplitude $6 \mu \mathrm{m}$ (which is higher than expected from ground modelling) needs to be added to the PR deformations;

- a sinusoidal deformation with 90-mm periodicity and $4 \mu \mathrm{m}$ amplitude along the symmetry plane needs to be added to the SR deformations.

2. There is no convincing evidence for the existence of additional "random"/Ruze-like distortion components on the surfaces.

3. Multi-mode effects qualitatively account for the high intensity of the grating lobes measured at 545 and $857 \mathrm{GHz}$, but cannot yet be used to provide detailed constraints.

4. The grating-related modifications to the reflector surfaces contain $0.12 \%$ power at $353 \mathrm{GHz}$ and $0.07 \%$ power at $217 \mathrm{GHz}$. 
Table 11. Residuals of RFFM2 for individual HFI detectors.

\begin{tabular}{|c|c|c|c|c|c|}
\hline \multirow[b]{2}{*}{ Detector } & \multirow{2}{*}{$\begin{array}{c}\text { Displacement }(\mathrm{mm}) \\
(\Delta x, \Delta y)\end{array}$} & \multicolumn{2}{|c|}{ Variance $(\mathrm{dB})$} & \multicolumn{2}{|c|}{$\operatorname{MPD}(\%)$} \\
\hline & & $\mathrm{a}$ & $\mathrm{b}$ & $\mathrm{a}$ & $\mathrm{b}$ \\
\hline $100-1 \ldots \ldots$ & $(-0.05,0.00)$ & 0.21 & 0.23 & 0.8 & 1.0 \\
\hline $100-2 \ldots \ldots$ & $(-0.05,0.08)$ & 0.27 & 0.25 & 1.6 & 1.5 \\
\hline $100-3 \ldots \ldots$ & $(-0.02,0.09)$ & 0.26 & 0.25 & 1.4 & 1.4 \\
\hline $100-4 \ldots \ldots$ & $(-0.12,0.09)$ & 0.19 & 0.19 & 1.0 & 1.0 \\
\hline $143-1 \ldots \ldots$ & $(0.01,-0.01)$ & 0.51 & 0.53 & 1.6 & 1.3 \\
\hline $143-2 \ldots \ldots$ & $(0.02,-0.09)$ & 0.38 & 0.39 & 1.5 & 1.2 \\
\hline $143-3 \ldots \ldots$ & $(0.01,-0.08)$ & 0.46 & 0.43 & 1.2 & 1.6 \\
\hline $143-4 \ldots \ldots$ & $(0.01,-0.08)$ & 0.64 & 0.63 & 1.4 & 1.6 \\
\hline $143-5 \ldots \ldots$ & $(0.00,-0.05)$ & & 0.52 & & 2.5 \\
\hline $143-6 \ldots . .$. & $(-0.02,-0.11)$ & & 0.40 & & 2.3 \\
\hline $143-7 \ldots \ldots$ & $(-0.04,-0.12)$ & & 0.43 & & 2.5 \\
\hline $217-1 \ldots \ldots$ & $(0.04,0.04)$ & & 0.36 & & 1.2 \\
\hline $217-2 \ldots \ldots$ & $(0.05,0.09)$ & & 0.37 & & 0.8 \\
\hline $217-3 \ldots \ldots$ & $(0.05,0.14)$ & & 0.39 & & 0.8 \\
\hline $217-4 \ldots \ldots$ & $(-0.04,0.23)$ & & 0.51 & & 0.4 \\
\hline $217-5 \ldots \ldots$ & $(0.08,0.07)$ & 0.52 & 0.57 & 1.5 & 1.4 \\
\hline $217-6 \ldots \ldots$ & $(0.03,0.00)$ & 0.43 & 0.44 & 1.5 & 1.6 \\
\hline $217-7 \ldots \ldots$ & $(0.00,0.11)$ & 0.46 & 0.46 & 1.3 & 1.4 \\
\hline $217-8 \ldots \ldots$ & $(0.06,0.11)$ & 0.48 & 0.49 & 1.7 & 1.4 \\
\hline $353-1 \ldots \ldots$ & $(0.09,0.02)$ & & 0.41 & & 1.4 \\
\hline $353-2 \ldots \ldots$ & $(0.07,-0.07)$ & & 0.56 & & 1.3 \\
\hline $353-3 \ldots \ldots$ & $(0.09,0.03)$ & 0.69 & 0.71 & 1.0 & 1.6 \\
\hline $353-4 \ldots \ldots$ & $(0.10,0.01)$ & 0.68 & 0.68 & 1.4 & 1.5 \\
\hline $353-5 \ldots \ldots$ & $(0.11,0.06)$ & 0.71 & 0.71 & 1.3 & 1.4 \\
\hline $353-6 \ldots \ldots$ & $(0.09,0.05)$ & 0.67 & 0.67 & 1.4 & 1.1 \\
\hline $353-7 \ldots \ldots$ & $(0.06,0.01)$ & & 0.56 & & 0.3 \\
\hline $353-8 \ldots \ldots$ & $(-0.03,-0.01)$ & & 0.40 & & 0.7 \\
\hline
\end{tabular}

Notes. MPD is the radial maximum of the integrated power difference between the measured and modelled beams.

\subsection{Retrieval of geometrical parameters}

Fitting of a large group of main beam shapes to GRASP models yields the following results.

1. Using only LFI beams allows us to retrieve a geometrical model that is mechanically within tolerances and provides a much better fit than the RFFM to the LFI beams measured in flight.

2. There are clear indications that the HFI fsbeams have significant deviations from "optical" beams; many of the fsbeams themselves contain suspicious features, and attempts at HFIonly retrieval yield mechanically unacceptable configurations. The implication is that the algorithm that generates the fsbeams adds non-optical features; however, the 2013 fsbeams appear to be less problematic in this sense than the 2015 fsbeams.

3. It is possible to retrieve a mechanically acceptable geometrical model using 2015 LFI beams and 2013 HFI fsbeams, with the only caveat being an apparent displacement in the $x$-direction of the HFI FPU with respect to the LFI FPU. This retrieval provides better performance than the RFFM for both LFI and HFI, although the LFI performance is slightly degraded with respect to that of the LFI-only retrieval.

\section{Conclusions}

Overall, the analysis of this paper confirms that the in-flight alignment of the main elements of the Planck optical telescope system was not significantly modified by stresses due to launch or cryogenic operations. Some deviations from expectations (as outlined below) were found in the shape of the reflectors. The sensitivity of the analysis did not allow us to evaluate potential changes in the properties over the duration of the mission.

The analysis of the grating lobes as measured in flight shows that the dimpling effect is significantly deeper than expected on the primary reflector, and that there are large-scale deformations on both reflectors, most probably caused by the reflector supports. Neither of these features were predicted from the pre-flight modelling. The amount of power in the grating lobes (approximately $0.1 \%$ at $217 \mathrm{GHz}$ ) is not insignificant compared to the uncertainties required for cosmological analysis of the Planck data and should be taken into account in future analysis. Shortcomings in the inputs to the mechanical model (Sect. 2.3) may be adequate to explain the poor prediction. It is clear that the small-scale mechanical behaviour of CFRP structures, such as that of the Planck reflectors, is more complex than expected.

The presence of significant time-response residuals in the "optical" HFI beams (fsbeams) has significantly limited their ability to constrain the best-fit geometrical model. In addition, the multi-moded nature of the $545-$ and $857 \mathrm{GHz}$ detectors has precluded their use in the retrieval of the geometry using the main beams, and allows only qualitative information to be extracted based on the grating lobes. At the lower frequencies, the LFI beams are very clean, but are relatively insensitive to the details of the geometry. It has not been possible to break some parameter degeneracies using the combined LFI and HFI data (mainly the average focal plane and reflector displacements in the axial direction). In spite of these shortcomings it has been possible, using flight data, to find a new geometrical model (RFFM2) that fits the in-flight data better than the pre-flight (RFFM) prediction, according to the metric that we have developed (the variance). The new geometrical model is physically reasonable, in the sense that it is within most of the estimated mechanical tolerances. However, the residual misalignment of individual LFI detectors is generally larger than expected from the pre-flight tolerance analysis. The model also indicates that the offsets between the LFI and HFI FPUs are slightly larger than expected in the $x$ and $z$ directions.

Overall, there remain significant uncertainties in the physical model of the main beams derived from this analysis (RFFM2), which are reflected in the remaining differences between the best-fit model and the planet measurements. These differences are of order $0.3 \%$ in integrated power in the main beam at $30 \mathrm{GHz}$, rising to about $1 \%$ at $70-100 \mathrm{GHz}$, then $2 \%$ at $143 \mathrm{GHz}$, and decreasing again to $1 \%$ at $353 \mathrm{GHz}$. One of the main factors contributing to these differences is the effect of the deconvolution of the time response from the HFI bolometer data.

The remaining differences between model and data are much larger than the uncertainties that are required for cosmological analysis of the Planck data. Therefore, the decision made early on to use the beam patterns measured in flight for the scientific analysis, limiting the use of GRASP predictions as much as possible, was clearly the right one. It is nonetheless inevitable that future experiments such as Planck will develop physical-optical models for at least some aspects of their analysis. We hope that the work described in this paper provides information that could help to decide to what extent they should rely on those models. Considering that one of the main factors limiting the fidelity of our model was the difficulty of separating the effects of time response from those of beam response in the HFI bolometers, future experiments should consider carefully how to decouple the uncertainties in the two effects. 
We also expect that in the future a much more rigorous methodology can be developed to implement a similar approach. Indeed, although we were able to find an adequate retrieval algorithm (i.e., the iterative sequence of optimizations with different sets of parameters), our iteration scheme needed a significant amount of manual tuning, requiring much trial and error. In addition there is no reliable way with our current method to obtain uncertainties in the parameters of the final retrieved model. Overall the analysis discussed here has been a very lengthy and computationally-intensive effort, and the final results have not been directly used in the Planck scientific pipeline. However, current and future enhancements in the computing speed of GRASP, and the recently-developed ability to deploy it on grid computing infrastructure, imply that it will be feasible to carry out many more GRASP simulations than was possible during this work. This should lead to the ability to run Monte-Carlotype sensitivity analysis based on GRASP, and multi-parameter Bayesian inference. Future efforts similar to the one described in this paper should consider developing such an approach.

Acknowledgements. This paper has been reviewed internally by the Planck Collaboration. A description of the Planck Collaboration and a list of its members, indicating which technical or scientific activities they have been involved in, can be found at http://wwW.cosmos.esa.int/web/planck/ planck-collaboration. The Planck Collaboration acknowledges the support of: ESA; CNES and CNRS/INSU-IN2P3-INP (France); ASI, CNR, and INAF (Italy); NASA and DoE (USA); STFC and UKSA (UK); CSIC, MICINN, and JA (Spain); Tekes, AoF, and CSC (Finland); DLR and MPG (Germany); CSA (Canada); DTU Space (Denmark); SER/SSO (Switzerland); RCN (Norway); SFI (Ireland); FCT/MCTES (Portugal); and DEISA (EU). The GRASP-based modelling work described here was carried out largely by Per Nielsen of TICRA, and benefitted from TICRA's long-standing, dedicated, and very productive involvement in the Planck mission covering, among others the specification, prediction, and characterization of the optical system of Planck.

\section{References}

Cuttaia, F., Terenzi, L., Morgante, G., et al. 2019, Exp. Astron., 47, 107

Dubruel, D. 2009, Planck Flight Radio Frequency Performances Quilting Analysis, Tech. Rep. H-P-ASP-AN-1839, Thales Alenia Space.

Jensen, F., Nielsen, P., Tauber, J., \& Martin-Polegre, A. 2010, Proceedings of EUCAP 2010

Murphy, J. A., Peacocke, T., Maffei, B., et al. 2010, J. Instrum., 5, T04001

Nielsen, P., Borries, O., Tauber, J., Martin-Polegre, A., \& Niels, H. B. 2011, Proc. EUCAP Ann. Symp., 1805

Nielsen, P. H. 2004, RF effect of dimpling distortion on the PLANCK telescope, Tech. Rep. S-1281-01, TICRA

Nielsen, P. H. 2005, Planck Telescope RF Performance Independent Verification Phase1, Tech. Rep. S-1287-07, TICRA

Planck HFI Core Team 2011, A\&A, 536, A4

Planck Collaboration II. 2011, A\&A, 536, A2

Planck Collaboration I. 2014, A\&A, 571, A1

Planck Collaboration II. 2014, A\&A, 571, A2

Planck Collaboration IV. 2014, A\&A, 571, A4

Planck Collaboration VI. 2014, A\&A, 571, A6

Planck Collaboration VII. 2014, A\&A, 571, A7

Planck Collaboration IX. 2014, A\&A, 571, A9
Planck Collaboration XV. 2014, A\&A, 571, A15

Planck Collaboration I. 2016, A\&A, 594, A1

Planck Collaboration II. 2016, A\&A, 594, A2

Planck Collaboration IV. 2016, A\&A, 594, A4

Planck Collaboration VII. 2016, A\&A, 594, A7

Planck Collaboration I. 2018, A\&A, submitted [arXiv:1807.06205]

Planck Collaboration Int. LII. 2017, A\&A, 607, A122

Roudier, G., Crill, B., Ducout, A., et al. 2015, in Proc. of the 36th ESA Antenna Workshop on Satellite Antenna Technology, ESA WPP-XXX

Tauber, J. A., Norgaard-Nielsen, H. U., Ade, P. A. R., et al. 2010, A\&A, 520, A2

${ }^{1}$ European Space Agency, ESTEC, Keplerlaan 1, 2201 AZ Noordwijk, The Netherlands e-mail: jtauber@cosmos.esa.int

2 APC, AstroParticule et Cosmologie, Université Paris Diderot, CNRS/IN2P3, CEA/lrfu, Observatoire de Paris, Sorbonne Paris Cité, 10, rue Alice Domon et Léonie Duquet, 75205 Paris Cedex 13, France

3 CNRS, IRAP, 9 Av. colonel Roche, BP 44346, 31028 Toulouse Cedex 4, France

${ }^{4}$ California Institute of Technology, Pasadena, CA, USA

5 DTU Space, National Space Institute, Technical University of Denmark, Elektrovej 327, DK-2800 Kgs. Lyngby, Denmark

${ }^{6}$ Departamento de Física, Universidad de Oviedo, C/ Federico García Lorca, 18, Oviedo, Spain

7 Department of Physics \& Astronomy, University of British Columbia, 6224 Agricultural Road, Vancouver, British Columbia, Canada

8 Department of Physics, Gustaf Hällströmin katu 2a, University of Helsinki, Helsinki, Finland

9 Department of Physics, Princeton University, Princeton, NJ, USA

10 Department of Physics, University of California, Santa Barbara CA, USA

11 Dipartimento di Fisica e Scienze della Terra, Università di Ferrara, Via Saragat 1, 44122 Ferrara, Italy

12 Haverford College Astronomy Department, 370 Lancaster Avenue, Haverford, PA, USA

13 Helsinki Institute of Physics, Gustaf Hällströmin katu 2, University of Helsinki, Helsinki, Finland

14 INAF, Istituto di Radioastronomia, Via Piero Gobetti 101, 40129 Bologna, Italy

15 INAF/IASF Bologna, Via Gobetti 101, Bologna, Italy

16 INFN, Sezione di Bologna, viale Berti Pichat 6/2, 40127 Bologna, Italy

17 Institut d'Astrophysique Spatiale, CNRS, Univ. Paris-Sud, Université Paris-Saclay, Bât. 121, 91405 Orsay Cedex, France

18 Jet Propulsion Laboratory, California Institute of Technology, 4800 Oak Grove Drive, Pasadena, CA, USA

19 LERMA, CNRS, Observatoire de Paris, 61 Avenue de l'Observatoire, Paris, France

20 The Oskar Klein Centre for Cosmoparticle Physics, Department of Physics, Stockholm University, AlbaNova, 10691 Stockholm, Sweden

21 Université de Toulouse, UPS-OMP, IRAP, 31028 Toulouse Cedex 4, France

22 TICRA, Laederstraede 34, Copenhaguen, Denmark 